TITLE:

\title{
Symmetry of the linearized Boltzmann equation and its application
}

$\operatorname{AUTHOR}(\mathrm{S}):$

TAKATA, Shigeru

CITATION:

TAKATA, Shigeru. Symmetry of the linearized Boltzmann equation and its application. Journal of Statistical Physics 2008, 136: 751-784

\section{ISSUE DATE:}

2008-12-18

URL:

http://hdl.handle.net/2433/77711

\section{RIGHT:}

The final publication is available at Springer via http://dx.doi.org/10.1007/s10955-0099793-2; この論文は出版社版でありません。引用の際には出版社版をご確認ご利用くだ さい。; This is not the published version. Please cite only the published version. 


\section{Kyoto University}

Graduate School of Engineering

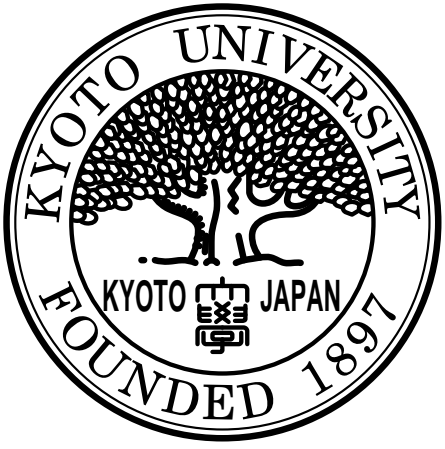

Preprint

\section{Symmetry of the linearized Boltzmann equation and its application}

\section{Shigeru TAKATA}

18 December 2008

(revised on 30 April 2009)

Department of Mechanical Engineering and Science 


\title{
Symmetry of the linearized Boltzmann equation and its application
}

\author{
Shigeru TAKATA
}

Received: / Accepted:

\begin{abstract}
A symmetric relation of macroscopic quantities between two different steady problems of the linearized Boltzmann equation is derived. A few applications to half-space problems are presented first. Then, for the gas in bounded or unbounded domains such that solid bodies or condensed phases are confined in a finite region, general representations of the mass, momentum, and heat fluxes through the boundary (possibly at a point on or on a part of it) are derived from the symmetric relation linked to the separability of boundary data. This result imply a reduction of the original problem to a single elemental problem in the same domain, as far as the fluxes are concerned. Many applications are also presented.
\end{abstract}

Keywords Boltzmann equation · reciprocity · Green function · Knudsen layer

PACS 51.10.+y $\cdot$ 47.45.-n $\cdot$ 05.20.Dd $\cdot 05.70 . \mathrm{Ln}$

Mathematics Subject Classification (2000) $76 \mathrm{P} 05 \cdot 82 \mathrm{~B} 40 \cdot 82 \mathrm{~B} 35$

\section{Introduction}

The linearized Boltzmann equation is widely used for the study of a slow rarefied gas flow or a gas in a micro scale system such as a micro channel, an aerosol particle, etc. One of the interesting feature in such gas systems is that fluid-dynamical and thermal phenomena are mutually inductive. For instance, a net heat flow through a channel is induced in the Poiseuille flow, while a net mass flow is induced by a temperature gradient along the wall (the thermal transpiration [1]). Those phenomena are sometimes related to each other, e.g., the former net flow is identical to the latter up to the dimensional factor in the above example [2]. The thermal polarization [3,4] and thermophoresis [5-7] of a particle are other examples of the mutually inductive phenomenon expected to be related to each other $[8,9]$. In the present paper, we investigate what kind of relation holds in general between two problems

S. Takata

Department of Mechanical Engineering and Science, also Advanced Research Institute of Fluid Engineering and Science, Graduate School of Engineering, Kyoto University, Kyoto 606-8501, Japan

Tel.: +81-75-7535802

Fax: +81-75-7534942

E-mail: takata@aero.mbox.media.kyoto-u.ac.jp 
described by the steady linearized Boltzmann equation. We will eventually derive a useful representation of the fluxes through the boundary for quite general situations.

First in Sect. 2, we consider two time-independent boundary-value problems of the linearized Boltzmann equation in the same domain. The domain may be bounded or unbounded, and its boundary is composed of two parts: one representing a simple solid surface or an interface with the condensed phase of the gas (the real boundary, for short) and the other representing an artificial boundary set in the interior of the gas, which includes the specular and periodic boundaries as a special case, or a far field in an unbounded domain (the imaginary boundary, for short). We derive a symmetric relation of averaged macroscopic quantities between the two problems by integrating both in space and molecular velocity a product of one solution and the other with reversed molecular velocity. The relation is obtained by the self-adjointness and parity of collision operator with the aid of the condition of detailed balance on the real boundary and its extension assumed on the artificial boundary, provided that the velocity distribution function approaches the given data sufficiently fast at a far distance for an unbounded domain. A concrete set of general situations in which the relation holds is also presented. These situations will be dealt with in the rest of the paper.

One of the situations where the symmetric relation holds is a spatially one-dimensional half-space problem of the linearized Boltzmann equation. In Sect. 3, we present some applications to half-space problems occurring in the study of the Knudsen layer structure [1012]. In Sects. 3.1 and 3.2 we show the recovery of known relations for the so-called slip and jump coefficients, while in Sect. 3.3 we provide unknown relations for jump coefficients and a couple of new numerical data by use of them. In the half-space problems, the boundary data on the real boundary affect the state of the gas in a far field. On the other hand, for bounded or unbounded domains such that the real boundary is confined in a finite region, we may deal with the boundary data separately, place by place, and consider a response of the system to elemental sources put on the boundary. We shall call the response the Green function in the present paper. In such domains, we can assure the symmetric relation to hold by a rather simple argument based on the Stokes set of equations. In Sect. 4, we present a fruitful consequence from the separability of the boundary data linked to the symmetric relation. To be specific, after a preliminary argument in Sect. 4.2, we present general expressions of the mass, momentum, and heat fluxes on the boundary in Sect. 4.3, which we call the representation theorem and is the second main outcome of the present paper. The obtained representation is not a mere superposition of the Green function. It tells that the problem of finding a flux through the boundary (possibly a part of or even a point of it) is reduced to finding the Green function for the elemental source corresponding to that flux put on the interested boundary. The elemental sources are a collision invariant distributed uniformly or linearly in space on the boundary (possibly on a part of or even at a point on it). Their correspondence to the fluxes will be clear in the course of discussion in Sects. 4.2 and 4.3. We also show corollaries of the theorem on the reciprocity of the fluxes induced by the Green functions in Sect. 4.4. This property will become important, especially when linked to the entropy theory to be developed in a separate paper. Various applications of the theorem will be shown in Sect. 5 .

Our Green function is not the ones discussed in $[13,14]$. The source is not in the equation but in the boundary condition as the macroscopically meaningful elemental data. This is intended to reflect the fact that the boundary data are given in accordance with the state of the surroundings at the macroscopic level. Our purpose is not to represent the solution itself as a superposition of the Green functions but to show the reduction of the original problem to obtaining a single elemental solution corresponding to the interested fluxes through the boundary. Simple analogues might come to mind. For instance, the Green reciprocity theo- 
rem [15-17] for the Laplace equation is known in the electrostatics for conductors and in the theory of heat conduction. For the continuum or near continuum gas (i.e., the system with small Knudsen numbers) in the linear regime, the flow field is described by the Stokes equation for the incompressible fluid and the temperature field by the Laplace equation [10-12]. From this point of view, the representation theorem may be regarded as the extension of the classical reciprocity to arbitrary Knudsen numbers, though it includes more. We will give a comment on this aspect in Corollary 9 in Sect. 5. We also mention a simple analogue in the theory of radiative transfer [18] to the example of a half-space problem in Sect. 3.2.

\section{Symmetry of the steady linearized Boltzmann equation}

\subsection{Problem}

We start with a physically rather abstract or seemingly artificial formulation of the problem.

Let us denote by $\boldsymbol{x}$ and $\boldsymbol{\zeta}$ the dimensionless position and molecular velocity. We shall consider a function $\phi(\boldsymbol{x}, \boldsymbol{\zeta})$ in a certain domain of $\boldsymbol{x}$, say $D$, and for the whole space of $\boldsymbol{\zeta}$ that satisfies the steady, inhomogeneous linearized Boltzmann equation:

$$
\zeta_{i} \frac{\partial \phi}{\partial x_{i}}+F_{i} \frac{\partial \phi}{\partial \zeta_{i}}=\frac{1}{K} \mathscr{L}(\phi)+I
$$

Here $\boldsymbol{F}$ is a given vector depending on $\boldsymbol{x}$ and $\boldsymbol{\zeta}$ and satisfies the condition $\frac{\partial}{\partial \zeta_{i}} F_{i} E=0$ with $E(\boldsymbol{\zeta})=\pi^{-3 / 2} \exp \left(-|\zeta|^{2}\right), K$ is an arbitrary positive constant $(0<K<\infty)$, and $I$ is a given function of $x$ and $\zeta$. The explicit form of the linearized collision integral $\mathscr{L}(\phi)$ is suppressed, because $\mathscr{L}$ is required only to have the following properties in the discussions:

(i) $\mathscr{L}(\cdot)$ commutes with the parity operator acting on $\zeta$ :

$$
\mathscr{L}(\Phi)^{-}=\mathscr{L}\left(\Phi^{-}\right) \text {for any } \Phi
$$

where the function with superscript ${ }^{-}$is defined as $\Psi^{-}(\boldsymbol{x}, \boldsymbol{\zeta}) \equiv \Psi(\boldsymbol{x},-\boldsymbol{\zeta})$.

(ii) $\mathscr{L}(\cdot)$ is self-adjoint:

$$
\langle\Phi \mathscr{L}(\Psi)\rangle=\langle\Psi \mathscr{L}(\Phi)\rangle \quad \text { for any } \Phi \text { and } \Psi,
$$

where the brackets $\langle\cdot\rangle$ indicate the following moment with respect to $\zeta$ :

$$
\langle\Phi\rangle=\int \Phi(\zeta) E(\zeta) \mathrm{d} \zeta
$$

(iii) $\mathscr{L}(\Phi)=0$ holds if and only if $\Phi$ is a linear combination of $1, \zeta$, and $|\zeta|^{2}$.

(iv) $\mathscr{L}(\cdot)$ is non-positive:

$$
\langle\Phi \mathscr{L}(\Phi)\rangle \leq 0 \text { for any } \Phi,
$$

and the equality holds if and only if $\Phi$ is a linear combination of $1, \boldsymbol{\zeta}$, and $|\boldsymbol{\zeta}|^{2}$.

The following obvious properties will be frequently used in the sequel:

$$
\left(\Phi^{-}\right)^{-}=\Phi, \quad\langle\Phi\rangle=\left\langle\Phi^{-}\right\rangle \quad \text { for any } \Phi .
$$

On a part of the boundary $\partial D$, which we denote by $\partial D_{\mathrm{w}}, \phi$ obeys the following condition:

$$
\phi=g_{\mathrm{w}}+\int_{\zeta_{n}^{*}<0} \frac{\left|\zeta_{n}^{*}\right| E\left(\boldsymbol{\zeta}^{*}\right)}{\left|\zeta_{n}\right| E(\boldsymbol{\zeta})} R\left(\boldsymbol{\zeta}^{*}, \boldsymbol{\zeta} ; \boldsymbol{x}\right)\left(\phi^{*}-g_{\mathrm{w}}^{*}\right) \mathrm{d} \boldsymbol{\zeta}^{*}, \quad \zeta_{\mathrm{n}}>0
$$


where $\zeta_{n}=\boldsymbol{\zeta} \cdot \boldsymbol{n}$ with $\boldsymbol{n}$ being the unit vector inward normal to the boundary $\partial D_{\mathrm{w}}$ at position $\boldsymbol{x}, g_{\mathrm{w}}$ is a given function of $\boldsymbol{x}$ and $\boldsymbol{\zeta}$ to be specified soon later in (5), and $\phi^{*}$ and $g_{\mathrm{w}}^{*}$ stand for $\phi\left(\boldsymbol{x}, \boldsymbol{\zeta}^{*}\right)$ and $g_{\mathrm{w}}\left(\boldsymbol{x}, \zeta^{*}\right)$ respectively. The kernel $R$ is a given function having the properties summarized in Appendix A, where and in what follows we denote by $R_{\mathrm{CR}}$ the kernel satisfying the condition $\left\langle\zeta_{n} \phi\right\rangle=0$ and by $R_{\mathrm{PR}}$ the kernel free from this condition. The required properties are different between $R_{\mathrm{CR}}$ and $R_{\mathrm{PR}}$. The function $g_{\mathrm{w}}(\boldsymbol{x}, \boldsymbol{\zeta})$ is defined by

$$
g_{\mathrm{w}}=\left\{\begin{array}{l}
2 \boldsymbol{\zeta} \cdot \boldsymbol{u}_{\mathrm{w}}+\left(|\boldsymbol{\zeta}|^{2}-\frac{5}{2}\right) \tau_{\mathrm{w}} \quad \text { if } R=R_{\mathrm{CR}}, \\
P_{\mathrm{w}}+2 \zeta \cdot \boldsymbol{u}_{\mathrm{w}}+\left(|\boldsymbol{\zeta}|^{2}-\frac{5}{2}\right) \tau_{\mathrm{w}} \quad \text { if } R=R_{\mathrm{PR}},
\end{array}\right.
$$

where $\boldsymbol{u}_{\mathrm{w}}, \tau_{\mathrm{w}}$, and $P_{\mathrm{w}}$ are given constants in $\boldsymbol{\zeta}$ with $\boldsymbol{u}_{\mathrm{w}} \cdot \boldsymbol{n}=0$. In general, $\boldsymbol{u}_{\mathrm{w}}, \tau_{\mathrm{w}}$, and $P_{\mathrm{w}}$ depend on the position $\boldsymbol{x}$ on $\partial D_{\mathrm{w}}$. Note that $g_{\mathrm{w}}$ is defined for the whole range of $\boldsymbol{\zeta}$.

Physically, $\partial D_{\mathrm{w}}$ is the part corresponding to the real boundary, which is the surface of a simple solid body (a simple surface, for short) or the interface with the condensed phase, i.e., liquid or solid, of the gas (the interface, for short). On the former, there occurs no mass flow across the boundary (i.e., $\left\langle\zeta_{n} \phi\right\rangle=0$ ), while on the latter it may occur in general. $R_{\mathrm{CR}}$ represents the reflection kernel for the former and $R_{\mathrm{PR}}$ that for the latter in the reference equilibrium state at rest. The deviation of the state of the boundary are represented by $P_{\mathrm{w}}$, $\boldsymbol{u}_{\mathrm{w}}$, and $\tau_{\mathrm{w}}$ involved in $g_{\mathrm{w}}$. The dependence of $R$ on $\boldsymbol{x}$ comes from the possible change of the boundary material and typically appears as the change of the accommodation coefficients.

From now on, we consider two functions $\phi^{A}$ and $\phi^{B}$ such that

1. $\phi^{A}$ satisfies (1) and (4) with $F_{i}=\mathscr{F}_{i}, I=I^{A}$, and $g_{\mathrm{w}}=g_{\mathrm{w}}^{A}$,

2. $\phi^{B}$ satisfies (1) and (4) with $F_{i}=\mathscr{F}_{i}^{-}, I=I^{B}$, and $g_{\mathrm{w}}=g_{\mathrm{w}}^{B}$,

where $R$ in (4) is common to $\phi^{A}$ and $\phi^{B}$. The aim of Sect. 2 is to derive a symmetric identity between the global quantities of $\phi^{A}$ and of $\phi^{B}$. For the moment, we proceed without any information about $\phi$ 's on the remaining part of the boundary $\partial D$, which we denote by $\partial D_{\mathrm{g}}$, i.e., $\partial D_{\mathrm{g}}=\partial D \backslash \partial D_{\mathrm{w}}$.

\subsection{Symmetric relation}

We first show a symmetric identity that can be obtained without specific information on $\partial D_{\mathrm{g}}$. The presented form is a slight extension of those in the literature (e.g., $\left.[13,19,20]\right)$ mainly in the sense that $I$ is arbitrary as far as the solution exists.

Proposition 1 Consider the functions $\phi^{A}$ and $\phi^{B}$ such that

(i) $\phi^{A}$ satisfies (1) and (4) with $F_{i}=\mathscr{F}_{i}, I=I^{A}$, and $g_{\mathrm{w}}=g_{\mathrm{w}}^{A}$,

(ii) $\phi^{B}$ satisfies (1) and (4) with $F_{i}=\mathscr{F}_{i}^{-}, I=I^{B}$, and $g_{\mathrm{w}}=g_{\mathrm{w}}^{B}$,

where $R$ in (4) is common to $\phi^{A}$ and $\phi^{B}$. If the kernel $R$ satisfies the condition of detailed balance $[13,21]$ (see Appendix $B$ ):

$$
\left|\zeta_{n}^{*}\right| R\left(\boldsymbol{\zeta}^{*}, \boldsymbol{\zeta} ; \boldsymbol{x}\right) E\left(\boldsymbol{\zeta}^{*}\right)=\left|\zeta_{n}\right| R\left(-\boldsymbol{\zeta},-\boldsymbol{\zeta}^{*} ; \boldsymbol{x}\right) E(\boldsymbol{\zeta}) \text { for } \boldsymbol{\zeta}_{n}>0, \zeta_{n}^{*}<0,
$$

the following symmetric identity holds:

$$
\begin{aligned}
\int_{\partial D_{\mathrm{w}}}\left\langle\zeta_{n} g_{\mathrm{w}}^{B-} \phi^{A}\right\rangle \mathrm{d} \mathrm{S}+ & \frac{1}{2} \int_{\partial D_{\mathrm{g}}}\left\langle\zeta_{n} \phi^{B-} \phi^{A}\right\rangle \mathrm{d} \mathrm{S}-\int_{D}\left\langle I^{B-} \phi^{A}\right\rangle \mathrm{d} \boldsymbol{x} \\
& =\int_{\partial D_{\mathrm{w}}}\left\langle\zeta_{n} g_{\mathrm{w}}^{A-} \phi^{B}\right\rangle \mathrm{d} \mathrm{S}+\frac{1}{2} \int_{\partial D_{\mathrm{g}}}\left\langle\zeta_{n} \phi^{A-} \phi^{B}\right\rangle \mathrm{d} \mathrm{S}-\int_{D}\left\langle I^{A-} \phi^{B}\right\rangle \mathrm{d} \boldsymbol{x},
\end{aligned}
$$

where $\mathrm{dS}$ is the surface element at position $\boldsymbol{x}$. 
Proof We first integrate (1) for $\phi^{A}$ (or $\phi^{B}$ ) multiplied by $\phi^{B-} E$ (or $\phi^{A-} E$ ) over the whole space of $\zeta$ :

$$
\begin{aligned}
\left\langle\zeta_{i} \frac{\partial \phi^{A}}{\partial x_{i}} \phi^{B-}\right\rangle+\left\langle\mathscr{F}_{i} \frac{\partial \phi^{A}}{\partial \zeta_{i}} \phi^{B-}\right\rangle & =\frac{1}{K}\left\langle\mathscr{L}\left(\phi^{A}\right) \phi^{B-}\right\rangle+\left\langle I^{A} \phi^{B-}\right\rangle \\
\left\langle\zeta_{i} \frac{\partial \phi^{B}}{\partial x_{i}} \phi^{A-}\right\rangle+\left\langle\mathscr{F}_{i}^{-} \frac{\partial \phi^{B}}{\partial \zeta_{i}} \phi^{A-}\right\rangle & =\frac{1}{K}\left\langle\mathscr{L}\left(\phi^{B}\right) \phi^{A-}\right\rangle+\left\langle I^{B} \phi^{A-}\right\rangle .
\end{aligned}
$$

Subtracting (8b) from (8a) leads to, with the aid of (2a), (2b), (3), and the condition for $\boldsymbol{F}$ immediately after (1),

$$
\frac{1}{2} \frac{\partial}{\partial x_{i}}\left\langle\zeta_{i} \phi^{B-} \phi^{A}\right\rangle+\left\langle I^{B-} \phi^{A}\right\rangle=\frac{1}{2} \frac{\partial}{\partial x_{i}}\left\langle\zeta_{i} \phi^{A-} \phi^{B}\right\rangle+\left\langle I^{A-} \phi^{B}\right\rangle
$$

Integrating (9) over the domain $D$ results in the well-known identity (e.g., see [13]):

$$
-\frac{1}{2} \int_{\partial D}\left\langle\zeta_{n} \phi^{B-} \phi^{A}\right\rangle \mathrm{dS}+\int_{D}\left\langle I^{B-} \phi^{A}\right\rangle \mathrm{dS}=-\frac{1}{2} \int_{\partial D}\left\langle\zeta_{n} \phi^{A-} \phi^{B}\right\rangle \mathrm{dS}+\int_{D}\left\langle I^{A-} \phi^{B}\right\rangle \mathrm{dS} .
$$

Next, we split the surface integrals into those on $\partial D_{\mathrm{w}}$ and $\partial D_{\mathrm{g}}$ and transform the integral on the former. Simple rearrangement yields on $\partial D_{\mathrm{w}}$

$$
\left\langle\zeta_{n} \phi^{B-} \phi^{A}\right\rangle=\left\langle\zeta_{n}\left(\phi^{B-}-g_{\mathrm{w}}^{B-}\right)\left(\phi^{A}-g_{\mathrm{w}}^{A}\right)\right\rangle+\left\langle\zeta_{n} g_{\mathrm{w}}^{B-} \phi^{A}\right\rangle+\left\langle\zeta_{n} \phi^{B-} g_{\mathrm{w}}^{A}\right\rangle-\left\langle\zeta_{n} g_{\mathrm{w}}^{B-} g_{\mathrm{w}}^{A}\right\rangle .
$$

The last term on the right-hand side vanishes because of (5) and $\boldsymbol{u}_{\mathrm{w}} \cdot \boldsymbol{n}=0$. Further, for $R$ satisfying the condition of detailed balance, the first term on the right-hand side vanishes, as shown in Appendix B. Thus, the integrand of the surface integral on $\partial D_{\mathrm{w}}$ is reduced to

$$
\left\langle\zeta_{n} \phi^{B-} \phi^{A}\right\rangle=\left\langle\zeta_{n} g_{\mathrm{w}}^{B-} \phi^{A}\right\rangle-\left\langle\zeta_{n} g_{\mathrm{w}}^{A-} \phi^{B}\right\rangle, \quad\left\langle\zeta_{n} \phi^{A-} \phi^{B}\right\rangle=\left\langle\zeta_{n} g_{\mathrm{w}}^{A-} \phi^{B}\right\rangle-\left\langle\zeta_{n} g_{\mathrm{w}}^{B-} \phi^{A}\right\rangle .
$$

Substitution into (10) yields the desired identity.

\subsubsection{Condition on $\partial D_{\mathrm{g}}$}

We now introduce the condition for $\phi$ on $\partial D_{\mathrm{g}}$ to refine Proposition 1 , and thus $\phi$ ought to be considered as a solution of the boundary-value problem of (1), (4), and the condition (11) on $\partial D_{\mathrm{g}}$ below. We shall consider three types of conditions on $\partial D_{\mathrm{g}}$ and correspondingly split it into three parts: $\partial D_{\mathrm{g}}^{(\mathrm{i})}, \partial D_{\mathrm{g}}^{(\mathrm{ii})}$, and $\partial D_{\mathrm{g}}^{(\mathrm{iii})}$. Each part is defined as follows.

(i) $\partial D_{\mathrm{g}}^{(\mathrm{i})}$ is the part where $\phi$ for the inward direction of $\zeta$ is given: ${ }^{1}$

$$
\phi(\boldsymbol{x}, \boldsymbol{\zeta})=h_{\mathrm{in}}(\boldsymbol{x}, \boldsymbol{\zeta}) \quad \text { for } \zeta_{n}>0, \boldsymbol{x} \in \partial D_{\mathrm{g}}^{(\mathrm{i})} .
$$

Here $h_{\text {in }}$ is a given function defined for $\zeta_{n}>0$. For later discussions, we extend the range of this function to the whole space of $\boldsymbol{\zeta}$ and denote the extended function by $h(\boldsymbol{x}, \boldsymbol{\zeta})$. The way of extension is arbitrary.

(ii) $\partial D_{\mathrm{g}}^{(i i)}$ is the part that reaches infinity when $D$ is an unbounded domain. The asymptotic form of $\phi$ for large $|x|$ is given:

$$
\phi(x, \boldsymbol{\zeta}) \rightarrow h(\boldsymbol{x}, \boldsymbol{\zeta}) \quad \text { as }|\boldsymbol{x}| \rightarrow \infty .
$$

Note that $h(\boldsymbol{x}, \boldsymbol{\zeta})$ is defined for the whole range of $\boldsymbol{\zeta}$ and satisfies (1) by definition.

\footnotetext{
1 Mathematically, the conditions (4) and (11a) do not exclude each other, so that there may be a part of the boundary which can be regarded as in $\partial D_{\mathrm{w}}$ or in $\partial D_{\mathrm{g}}^{(\mathrm{i})}$. However, there arises no difference to be cared.
} 
(iii) $\partial D_{\mathrm{g}}^{(\mathrm{iii})}$ is the part where $\phi$ for the inward direction of $\zeta$ at position $x$ is related to that for the outward direction of $\boldsymbol{\zeta}^{\prime}$ at position $\boldsymbol{x}^{\prime}$ :

$$
\phi(\boldsymbol{x}, \boldsymbol{\zeta})=h(\boldsymbol{x}, \boldsymbol{\zeta})+\int_{\partial D_{\mathrm{g}}^{(\mathrm{iii})}} \int_{\zeta_{n^{\prime}}^{\prime}<0} P\left(\boldsymbol{x}^{\prime}, \boldsymbol{\zeta}^{\prime}, \boldsymbol{x}, \boldsymbol{\zeta}\right)\left(\phi^{\prime}-h^{\prime}\right) \mathrm{d} \boldsymbol{\zeta}^{\prime} \mathrm{d} \mathrm{S}^{\prime} \quad \text { for } \zeta_{n}>0, \boldsymbol{x} \in \partial D_{\mathrm{g}}^{(\mathrm{iii})},
$$

where $h(\boldsymbol{x}, \boldsymbol{\zeta})$ is a given function defined for the whole range of $\boldsymbol{\zeta}$ and for any $\boldsymbol{x} \in \partial D_{\mathrm{g}}^{(\mathrm{iii})}$, $\zeta_{n^{\prime}}^{\prime}=\boldsymbol{\zeta}^{\prime} \cdot \boldsymbol{n}^{\prime}$, and $\boldsymbol{n}^{\prime}$ and $\mathrm{d} \mathrm{S}^{\prime}$ are the inward unit vector normal to $\partial D_{\mathrm{g}}^{(\mathrm{iii})}$ and the surface element at point $\boldsymbol{x}^{\prime}$ respectively. $\boldsymbol{\phi}^{\prime}$ and $h^{\prime}$ stand for $\phi\left(\boldsymbol{x}^{\prime}, \boldsymbol{\zeta}^{\prime}\right)$ and $h\left(\boldsymbol{x}^{\prime}, \boldsymbol{\zeta}^{\prime}\right)$ respectively. $P$ is a given function defined for $\zeta_{n}>0$ and $\zeta_{n^{\prime}}^{\prime}<0$ that prescribes the relation between two points $\boldsymbol{x}$ and $\boldsymbol{x}^{\prime}$ on $\partial D_{\mathrm{g}}^{(\mathrm{iii})}$. Here we restrict $P$ to the functions that meet the following conditions (an extension of the conditions in Appendices A and B):

(a) For $\zeta_{n}>0$ and $\zeta_{n^{\prime}}^{\prime}<0$,

$$
\left|\zeta_{n}\right| E(\boldsymbol{\zeta}) P\left(\boldsymbol{x}^{\prime}, \boldsymbol{\zeta}^{\prime}, \boldsymbol{x}, \boldsymbol{\zeta}\right)=\left|\zeta_{n^{\prime}}^{\prime}\right| E\left(\boldsymbol{\zeta}^{\prime}\right) P\left(\boldsymbol{x},-\boldsymbol{\zeta}, \boldsymbol{x}^{\prime},-\boldsymbol{\zeta}^{\prime}\right)
$$

(b) For a certain given function $g_{0}(\boldsymbol{x}, \boldsymbol{\zeta}) \geq 0$ defined for $\zeta_{n}>0$ and $\boldsymbol{x} \in \partial D_{\mathrm{g}}^{(\mathrm{iii})}$, the following relation holds:

$$
1=g_{0}(\boldsymbol{x}, \boldsymbol{\zeta})+\int_{\partial D_{\mathrm{g}}^{(\mathrm{iii})}} \int_{\zeta_{n^{\prime}}<0} P\left(\boldsymbol{x}^{\prime}, \boldsymbol{\zeta}^{\prime}, \boldsymbol{x}, \boldsymbol{\zeta}\right) \mathrm{d} \boldsymbol{\zeta}^{\prime} \mathrm{d} \mathrm{S}^{\prime} \quad \text { for } \zeta_{n}>0
$$

(c) $P\left(\boldsymbol{x}^{\prime}, \boldsymbol{\zeta}^{\prime}, \boldsymbol{x}, \boldsymbol{\zeta}\right) \geq 0$, and it is not identically zero.

The property (a) is an extension of the condition of detailed balance to among different points on the boundary. It should be noted that the uniqueness condition corresponding to the third property of $R_{\mathrm{CR}}$ and $R_{\mathrm{CR}}$ in Appendix $\mathrm{A}$ is not required of $P$. Thus, the specular and periodic type boundaries are classified into $\partial D_{\mathrm{g}}^{(\mathrm{iii})}$. Due to the property (c), (11a) is excluded from (11c).

Some physical comments would be in order on the boundary conditions. As mentioned before, $\partial D_{\mathrm{w}}$ is the part corresponding to the real boundary, i.e., a simple surface or the interface. On the former, there occurs no mass flow across the boundary, while on the latter it may occur in general. The kernel $R_{\mathrm{CR}}$ represents the reflection rule on the former and $R_{\mathrm{PR}}$ on the latter. It is important to notice that the specular reflection condition is excluded from the part $\partial D_{\mathrm{w}}$ because of the third property of $R_{\mathrm{CR}}$ and $R_{\mathrm{PR}}$ in Appendix A. The remaining part $\partial D_{\mathrm{g}}$ is the imaginary boundary, which is set inside a gas. The specular reflection and periodic boundary conditions are a typical example of the part $\partial D_{\mathrm{g}}^{(\mathrm{iii})}$. It is readily checked that they have the properties required of $P$. Arbitrariness of $h$ on $\partial D_{\mathrm{g}}^{(\mathrm{i})}$ and $\partial D_{\mathrm{g}}^{(\mathrm{iii})}$ aims at the application to a rather artificial problem setting often adopted in numerical simulations.

We close the present subsection with the following lemma:

Lemma 1 Let $\phi^{A}$ and $\phi^{B}$ obey the condition (11) with $h=h^{A}$ and $h=h^{B}$ respectively, where $P$ in $(11 \mathrm{c})$ is common to $\phi^{A}$ and $\phi^{B}$. Then the following identity holds:

$$
\int_{\partial D_{\mathrm{g}}^{(\mathrm{i})} \cup \partial D_{\mathrm{g}}^{(\mathrm{iii})}}\left\langle\zeta_{n}\left(\phi^{A-}-h^{A-}\right)\left(\phi^{B}-h^{B}\right)\right\rangle \mathrm{d} \mathrm{S}=0,
$$

where $h^{A}$ and $h^{B}$ on $\partial D_{\mathrm{g}}^{(\mathrm{i})}$ are an arbitrary extension of $h_{\mathrm{in}}^{A}$ and $h_{\mathrm{in}}^{B}$ to the whole range of $\zeta$. 
京都大学

A Self-archived copy in

KYOTO UNIVERSITY

Kyoto University Research Information Repository

KUTENAI In 工

7

Proof For brevity, we denote $\phi^{\alpha}-h^{\alpha}$ by $\psi^{\alpha}(\alpha=A, B)$. Obviously $\psi^{\alpha}=0$ for $\zeta_{n}>0$ on $\partial D_{\mathrm{g}}^{(\mathrm{i})}$. The integrand on $\partial D_{\mathrm{g}}^{(\mathrm{i})}$ is readily seen to vanish by a change of variables:

$$
\left\langle\zeta_{n} \psi^{A-} \psi^{B}\right\rangle=\int_{\zeta_{n}>0} \zeta_{n} \psi^{A-} \psi^{B} E \mathrm{~d} \zeta-\int_{\zeta_{n}>0} \zeta_{n} \psi^{A} \psi^{B-} E \mathrm{~d} \zeta=0
$$

and (12) is reduced to the surface integral on $\partial D_{\mathrm{g}}^{(\mathrm{iii})}$. For the reduced integral

$$
\int_{\partial D_{\mathrm{g}}^{(\mathrm{iii})}}\left\langle\zeta_{n} \psi^{A-} \psi^{B}\right\rangle \mathrm{dS}=\int_{\partial D_{\mathrm{g}}^{(\mathrm{iii})}} \int_{\zeta_{n}>0} \zeta_{n} \psi^{A-} \psi^{B} E \mathrm{~d} \zeta \mathrm{d} S-\int_{\partial D_{\mathrm{g}}^{(i i i)}} \int_{\zeta_{n}>0} \zeta_{n} \psi^{A} \psi^{B-} E \mathrm{~d} \zeta \mathrm{dS}
$$

we use (11c) for $\psi^{B}$ in the first term and that for $\psi^{A}$ in the second term on the right-hand side. Then, by the use of the property (a) of $P$, the first and second terms are seen to cancel out each other in a way similar to the proof of Lemma 4 in Appendix B.

2.2.2 Symmetric relation

We now present the first main outcome of the present paper, which is the refinement of Proposition 1 due to Lemma 1:

Proposition 2 (symmetric relation) Consider the solutions $\phi^{A}$ and $\phi^{B}$ of the boundaryvalue problem (1), (4), and (11) such that

(i) $\phi^{A}$ is a solution in the case of $I=I^{A}, g_{\mathrm{w}}=g_{\mathrm{w}}^{A}, F_{i}=\mathscr{F}_{i}$, and $h=h^{A}$,

(ii) $\phi^{B}$ is a solution in the case of $I=I^{B}, g_{\mathrm{w}}=g_{\mathrm{w}}^{B}, F_{i}=\mathscr{F}_{i}^{-}$, and $h=h^{B}$,

where $R$ in (4) and $P$ in (11c) are common to the problems. Then,

1. If the kernel $R$ on $\partial D_{\mathrm{w}}$ satisfies the condition of detailed balance (6),

2. and if the part $\partial D_{\mathrm{g}}^{(\mathrm{ii})}$ is absent or $\phi^{A}$ and $\phi^{B}$ approach $h^{A}$ and $h^{B}$ sufficiently fast in (11b) so that

$$
\int_{\partial D_{\mathrm{g}}^{(\mathrm{iii})}}\left\langle\zeta_{n}\left(\phi^{A-}-h^{A-}\right)\left(\phi^{B}-h^{B}\right)\right\rangle \mathrm{dS}=0
$$

the following equality holds:

$$
\begin{aligned}
& \int_{\partial D_{\mathrm{w}}}\left\langle\zeta_{n} g_{\mathrm{w}}^{B-} \phi^{A}\right\rangle \mathrm{d} \mathrm{S}+\int_{\partial D_{\mathrm{g}}}\left\langle\zeta_{n} h^{B-} \phi^{A}\right\rangle \mathrm{dS}-\frac{1}{2} \int_{\partial D_{\mathrm{g}}}\left\langle\zeta_{n} h^{B-} h^{A}\right\rangle \mathrm{dS}-\int_{D}\left\langle I^{B-} \phi^{A}\right\rangle \mathrm{d} \boldsymbol{x} \\
= & \int_{\partial D_{\mathrm{w}}}\left\langle\zeta_{n} g_{\mathrm{w}}^{A-} \phi^{B}\right\rangle \mathrm{d} \mathrm{S}+\int_{\partial D_{\mathrm{g}}}\left\langle\zeta_{n} h^{A-} \phi^{B}\right\rangle \mathrm{d} \mathrm{S}-\frac{1}{2} \int_{\partial D_{\mathrm{g}}}\left\langle\zeta_{n} h^{A-} h^{B}\right\rangle \mathrm{d} \mathrm{S}-\int_{D}\left\langle I^{A-} \phi^{B}\right\rangle \mathrm{d} \boldsymbol{x} .
\end{aligned}
$$

It should be reminded that $h^{A}$ and $h^{B}$ on $\partial D_{\mathrm{g}}^{(\mathrm{i})}$ may be any extension of $h_{\mathrm{in}}^{A}$ and $h_{\mathrm{in}}^{B}$ to the whole range of $\zeta$ [see (i) in Sect. 2.2.1]. ${ }^{2}$

2 The respective values of the second and third terms of each side of (14) depend on the way of extension of $h_{\mathrm{in}}^{A}$ and $h_{\mathrm{in}}^{B}$. However, the following relation always holds:

$$
\int_{\partial D_{\mathrm{g}}}\left\langle\zeta_{n} h^{B-} \phi^{A}\right\rangle \mathrm{dS}-\frac{1}{2} \int_{\partial D_{\mathrm{g}}}\left\langle\zeta_{n} h^{B-} h^{A}\right\rangle \mathrm{dS}=\int_{\partial D_{\mathrm{g}}}\left\langle\zeta_{n} h^{A-} \phi^{B}\right\rangle \mathrm{dS}-\frac{1}{2} \int_{\partial D_{\mathrm{g}}}\left\langle\zeta_{n} h^{A-} h^{B}\right\rangle \mathrm{dS} .
$$


Proof The refinement is achieved by the reduction of $\left\langle\zeta_{n} \phi^{A-} \phi^{B}\right\rangle$ on $\partial D_{\mathrm{g}}$ due to Lemma 1:

$$
\begin{aligned}
\int_{\partial D_{\mathrm{g}}}\left\langle\zeta_{n} \phi^{A-} \phi^{B}\right\rangle \mathrm{dS}=\int_{\partial D_{\mathrm{g}}}\left(\left\langle\zeta_{n} h^{A-} \phi^{B}\right\rangle+\left\langle\zeta_{n} \phi^{A-} h^{B}\right\rangle-\left\langle\zeta_{n} h^{A-} h^{B}\right\rangle\right) \mathrm{dS} & \\
& +\int_{\partial D_{\mathrm{g}}^{(i i)}}\left\langle\zeta_{n}\left(\phi^{A-}-h^{A-}\right)\left(\phi^{B}-h^{B}\right)\right\rangle \mathrm{d} \mathrm{S} .
\end{aligned}
$$

It should be noted that $I$ and $h$ are not restricted to any specific form as far as the solution $\phi$ exists (see the example in Sect. 3.3).

The condition (13) is fulfilled for bounded domains. It is also fulfilled for unbounded domains at least in the following two general situations for $\boldsymbol{F}=0$ :

1. $D$ is a spatially one-dimensional half-space.

2. $D$ is an unbounded three dimensional domain and $\partial D_{\mathrm{w}}$ is confined in a finite region, so that $\partial D_{\mathrm{g}}=\partial D_{\mathrm{g}}^{(\mathrm{ii})}$.

The first case is obvious because the area of surface integral does not change in passing to the limit $|\boldsymbol{x}| \rightarrow \infty$, and $\phi^{A}$ and $\phi^{B}$ in the surface integral on $\partial D_{\mathrm{g}}$ may be replaced by $h^{A}$ and $h^{B}$. The second case is not trivial and is due to Lemma 2 to be shown soon later. ${ }^{3}$

In the rest of the paper, we work on bounded domains and unbounded domains in the situations raised above. We first present some applications to the first case, i.e., onedimensional half-space problems, in Sect. 3. The first case is not merely simple but also requires a separate discussion from the second case. It is due to the fact that the source on the real boundary $g_{\mathrm{w}}$ does affect the state of the gas in a far field in that case and thus $h$ cannot be given independent of $g_{\mathrm{w}}$. [22] In contrast, we may separately deal with the sources on the boundaries in the second case, i.e., the three dimensional unbounded domain, and consequently can establish a unified theoretical framework with the case of bounded domain. We present this unified approach in Sect. 4, which leads to the second main outcome of the present paper on general representations of the mass, momentum, and heat fluxes passing through the boundary. We will show its applications in Sect. 5.

We close the present subsection with the announced lemma:

Lemma 2 Let $\phi^{A}$ and $\phi^{B}$ satisfy (1) with $\boldsymbol{F}=0$ in an unbounded domain $D$ with $\partial D_{\mathrm{g}}=$ $\partial D_{\mathrm{g}}^{(\mathrm{ii})}$, where $h=h^{A}$ and $h^{B}$ respectively in $(11 \mathrm{~b})$. Then the following equality holds:

$$
\int_{\partial D_{\mathrm{g}}}\left\langle\zeta_{n}\left(\phi^{A-}-h^{A-}\right)\left(\phi^{B}-h^{B}\right)\right\rangle \mathrm{dS}=0
$$

Proof Let us denote $\phi^{\alpha}-h^{\alpha}$ by $\psi^{\alpha}(\alpha=A, B)$. Since, by definition, $h^{\alpha}$ is a solution of $(1)^{4}$ with $\boldsymbol{F}=0, \psi^{\alpha}$ is a solution of (1) with $\boldsymbol{F}=0$ and $I^{\alpha}=0$ and tends to vanish as $|\boldsymbol{x}| \rightarrow \infty$. Now consider a sufficiently large sphere that contains the real boundary $\partial D_{\mathrm{w}}$ inside. We denote by $S_{\mathrm{g}}$ the surface of the sphere and by $r_{\mathrm{g}}$ its radius. $\psi^{\alpha}$ varies with the scale of $r_{\mathrm{g}}$ near $S_{\mathrm{g}}$ for sufficiently large $r_{\mathrm{g}}$, and the behavior at a far distance is well described by the Grad-Hilbert expansion [10] for small $\mathrm{Kn} / r_{\mathrm{g}}(\ll 1)$. This implies that the flow velocity and the pressure due to $\psi^{\alpha}$ are described by the Stokes equation for the incompressible fluid, while the temperature due to $\psi^{\alpha}$ is described by the Laplace equation [10]. Thanks to the

\footnotetext{
3 As is clear from the proof of Lemma 2, the key estimate (33) in [19] is incorrect.

4 In general, $h^{\alpha}$ may have singularities in the domain under consideration, so does $\psi^{\alpha}$. However, it does not cause any trouble, because only the outer region, where $h^{\alpha}$ and $\psi^{\alpha}$ are regular, will be considered.
} 


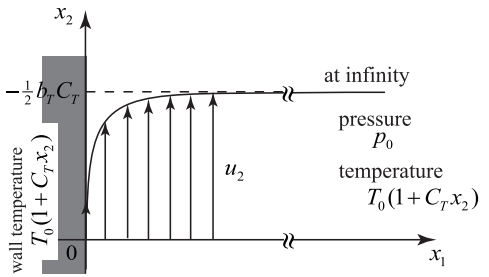

(a) Thermal creep

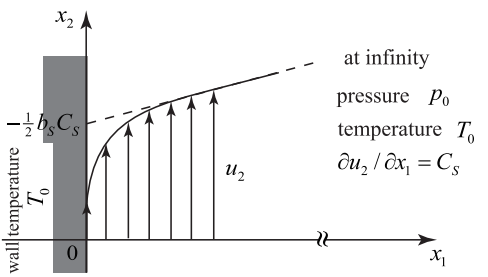

(b) Shear flow

Fig. 1 Thermal creep and shear flow problems.

general solution of the Stokes equation [23-25] and the property of the harmonic functions (e.g., [26]), the pressure due to $\psi^{\alpha}$ is seen to be of $O\left(|x|^{-2}\right)$, while the flow velocity and temperature due to $\psi^{\alpha}$ are seen to be of $O\left(|\boldsymbol{x}|^{-1}\right)$. Thus $\psi^{\alpha}$ for large $|\boldsymbol{x}|$ is estimated as

$$
\psi^{\alpha}=2 \zeta_{i} c_{i}^{\alpha}+\left(|\boldsymbol{\zeta}|^{2}-\frac{5}{2}\right) c^{\alpha}+O\left(|x|^{-2}\right) \quad(\alpha=A, B),
$$

where $c_{i}^{\alpha}$ and $c^{\alpha}$ are a quantity of $O\left(|\boldsymbol{x}|^{-1}\right)$, independent of $\zeta$, and we have

$$
\int_{\partial D_{\mathrm{g}}}\left\langle\zeta_{n} \psi^{A-} \psi^{B}\right\rangle \mathrm{dS}=\lim _{r_{\mathrm{g}} \rightarrow \infty} \int_{\partial S_{\mathrm{g}}}\left\langle\zeta_{n} \psi^{A-} \psi^{B}\right\rangle \mathrm{dS}=\lim _{r_{\mathrm{g}} \rightarrow \infty} \int_{\partial S_{\mathrm{g}}} O\left(|\boldsymbol{x}|^{-3}\right) \mathrm{dS}=0,
$$

which is the desired equality.

\section{Application to half-space problems}

We show some applications of the symmetric relation (14) to half-space problems. Throughout this section, the real boundary is assumed to be locally isotropic [12,10]. Sections 3.1 and 3.2 present the recovery of known relations, while Sect. 3.3 provides unknown relations.

\subsection{Shear and thermal creep flows over a plane wall}

When a rarefied gas is bounded by a wall with a gradient of temperature along its surface, a flow is induced along the wall in the direction of the gradient (the thermal creep flow; see, e.g., [1,27-29]). We discuss a cross relation of this flow to the shear flow over the wall.

Let us denote by $x_{i} L$ the space coordinate with $L=(\sqrt{\pi} / 2) \ell_{0}$ where $\ell_{0}$ is the mean free path of a molecule at the equilibrium state at rest with pressure $p_{0}$ and temperature $T_{0}$. Consider a gas occupying a half space $\left(x_{1}>0\right)$ over a resting plane wall in the following two situations depicted in Fig. 1:

1. Thermal creep (problem T, for short): The temperature of the wall is given by $T_{0}(1+$

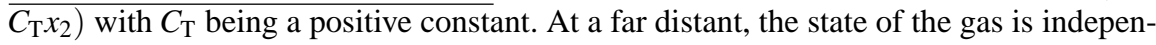
dent of $x_{1}$, the pressure is $p_{0}$, and the temperature is the same as that of the wall.

2. Shear flow (problem $\mathrm{S}$, for short): The temperature of the wall is $T_{0}$. At a far distance, the gas pressure is $p_{0}$, the temperature is $T_{0}$, and the $x_{2}$-component of the flow changes linearly in $x_{1}$ with a constant gradient $\left[\left(2 k T_{0} / m\right)^{1 / 2} / L\right] C_{\mathrm{S}}$, where $k$ and $m$ is the Boltzmann constant and the mass of a molecule, respectively. 
The condition $C_{\mathrm{T}},\left|C_{\mathrm{S}}\right| \ll 1$ is assumed for linearization. We denote by $2 p_{0}\left(2 k T_{0} / m\right)^{-5 / 2}(1+$ $\left.\phi_{J}\right) E$ the velocity distribution function for problem $J(J=\mathrm{T}, S) . \phi_{J}$ is known to be sought in the form of $\phi_{\mathrm{T}}=C_{\mathrm{T}}\left[\left(|\zeta|^{2}-\frac{5}{2}\right) x_{2}-\Phi_{\mathrm{T}}\left(x_{1}, \zeta\right)\right]$ and $\phi_{\mathrm{S}}=C_{\mathrm{S}}\left[2 \zeta_{2} x_{1}-\Phi_{\mathrm{S}}\left(x_{1}, \zeta\right)\right]$, where $\Phi_{J}$ is a solution of the following boundary-value problem:

$$
\begin{aligned}
\zeta_{1} \frac{\partial \Phi_{J}}{\partial x_{1}} & =\mathscr{L}\left(\Phi_{J}\right)+I_{J} \quad(J=\mathrm{T}, \mathrm{S}), \\
\Phi_{J} & =\int_{\zeta_{1}^{*}<0} \frac{\left|\zeta_{1}^{*}\right| E\left(\boldsymbol{\zeta}^{*}\right)}{\zeta_{1} E(\boldsymbol{\zeta})} R_{\mathrm{CR}}\left(\boldsymbol{\zeta}^{*}, \zeta\right) \phi_{J}^{*} \mathrm{~d} \boldsymbol{\zeta}^{*}, \quad \zeta_{1}>0, x_{1}=0, \\
\Phi_{J} & \rightarrow h_{J} \text { as } x_{1} \rightarrow \infty,
\end{aligned}
$$

where

$$
I_{\mathrm{T}}=\zeta_{2}\left(|\zeta|^{2}-\frac{5}{2}\right), \quad h_{\mathrm{T}}=b_{\mathrm{T}} \zeta_{2}+\zeta_{2} A(|\zeta|), \quad I_{\mathrm{S}}=2 \zeta_{1} \zeta_{2}, \quad h_{\mathrm{S}}=b_{\mathrm{S}} \zeta_{2}+\zeta_{1} \zeta_{2} B(|\zeta|)
$$

$R_{\mathrm{CR}}$ is independent of $\boldsymbol{x}, A(|\boldsymbol{\zeta}|)$ is the solution of $\mathscr{L}\left(\zeta_{i} A\right)=-\zeta_{i}\left(|\boldsymbol{\zeta}|^{2}-\frac{5}{2}\right)$ such that $\left\langle|\boldsymbol{\zeta}|^{2} A\right\rangle=$ 0 , and $B(|\zeta|)$ is the solution of $\mathscr{L}\left(\zeta_{i j} B\right)=-2 \zeta_{i j}$ with $\zeta_{i j}=\zeta_{i} \zeta_{j}-\frac{1}{3}|\zeta|^{2} \delta_{i j}$.

The reduced problem is known to have a solution if and only if $b_{J}$ takes a special value, and the solution is unique and approaches $h_{J}$ exponentially fast as $x_{1} \rightarrow \infty[22,30-33]$. With these properties in mind, we use Proposition 2 by putting $\phi^{A}=\Phi_{\mathrm{T}}$ and $\phi^{B}=\Phi_{\mathrm{S}}$ to have

$$
-\frac{1}{2}\left\langle\zeta_{1} h_{\mathrm{S}}^{-} h_{\mathrm{T}}\right\rangle-\int_{0}^{\infty}\left\langle I_{\mathrm{S}}^{-} \Phi_{\mathrm{T}}\right\rangle \mathrm{d} x_{1}=-\frac{1}{2}\left\langle\zeta_{1} h_{\mathrm{T}}^{-} h_{\mathrm{S}}\right\rangle-\int_{0}^{\infty}\left\langle I_{\mathrm{T}}^{-} \Phi_{\mathrm{S}}\right\rangle \mathrm{d} x_{1}
$$

In the meantime, the integration of (15a) for $J=\mathrm{T}$ multiplied by $\zeta_{2} E$ shows the second term on the left-hand side to vanish, and we eventually obtain

$$
\left\langle\zeta_{1}^{2} \zeta_{2}^{2} B(|\zeta|)\right\rangle b_{\mathrm{T}}=-\int_{0}^{\infty}\left\langle\zeta_{2}\left(|\zeta|^{2}-\frac{5}{2}\right) \Phi_{\mathrm{S}}\right\rangle \mathrm{d} x_{1}-\left\langle\zeta_{1}^{2} \zeta_{2}^{2} A(|\zeta|) B(|\zeta|)\right\rangle .
$$

As depicted in Fig. 1(a), $-\frac{1}{2} b_{\mathrm{T}}$ is the dimensionless flow velocity at a far distance in the $x_{2}$-direction normalized by $C_{\mathrm{T}}$ in problem $\mathrm{T}$, while $\left\langle\zeta_{2}\left(|\zeta|^{2}-\frac{5}{2}\right) \Phi_{\mathrm{S}}\right\rangle$ is the dimensionless heat flow in the same direction normalized by $C_{\mathrm{S}}$ in problem $\mathrm{S}$. Equation (16) represents the cross relation between the two problems. Incidentally, $2\left\langle\zeta_{1}^{2} \zeta_{2}^{2} B(|\zeta|)\right\rangle$ is the dimensionless viscosity and $2\left\langle\zeta_{1}^{2} \zeta_{2}^{2} A(|\zeta|) B(|\zeta|)\right\rangle$ the dimensionless coefficient of the thermal stress.

The relation (16) can be checked by the numerical data in the literature (see, e.g., [20]). By using the notation in [10], (16) is rewritten as

$$
\gamma_{1} K_{1}=-\frac{1}{2} \gamma_{3}+\int_{0}^{\infty} H_{A}(\eta) \mathrm{d} \eta
$$

where $\gamma_{1}=2\left\langle\zeta_{1}^{2} \zeta_{2}^{2} B\right\rangle, K_{1}=b_{\mathrm{T}} / 2, \gamma_{3}=2\left\langle\zeta_{1}^{2} \zeta_{2}^{2} A B\right\rangle, H_{A}=-\left\langle\zeta_{2}\left(|\zeta|^{2}-\frac{5}{2}\right) \Phi_{\mathrm{S}}\right\rangle$, and $\eta=x_{1}$. For the diffuse reflection boundary condition, $K_{1}$ and $H_{A}$ have been computed accurately as

$$
\begin{aligned}
& K_{1}=-0.38316, \quad \int_{0}^{\infty} H_{A}(\eta) \mathrm{d} \eta=\frac{1}{2} \times 0.23368 \quad(\text { BGK model }[27,34,10]), \\
& \left.K_{1}=-0.6465, \quad \int_{0}^{\infty} H_{A}(\eta) \mathrm{d} \eta=0.1530 \quad \text { (Boltzmann equation; hard sphere }[28,10]\right) .
\end{aligned}
$$

For the latter, we show the values recomputed from the data obtained in [35] ( $K_{1}=-0.6463$ and $\int_{0}^{\infty} H_{A} \mathrm{~d} \eta$ is not given in [10,28]). According to [10], both $\gamma_{1}$ and $\gamma_{3}$ are unity for the BGK $^{5}$ model, while $\gamma_{1}=1.270042427$ and $\gamma_{3}=1.947906335$ for the hard-sphere Boltzmann equation. Substitution of these values shows the relation (16) or (17) to hold.

\footnotetext{
5 The BGK (Bhatnagar-Gross-Krook) model is termed "BKW" (Boltzmann-Krook-Welander) equation in the cited references because of the independent contribution by Welander.
} 
3.2 Evaporation and condensation on a plane condensed phase

Consider a gas occupying a half space bounded by its plane condensed phase. The condensed phase is at rest and its temperature is uniform and constant. At a far distance, the gas is in the state of uniform pressure with uniform flow and temperature gradient normal to the interface. We discuss the steady behavior of the gas in the situation (see, e.g., [36-38]).

We use the same notation as Sect. 3.1 and take the temperature of the condensed phase and the corresponding saturation gas pressure as the reference temperature $T_{0}$ and pressure $p_{0}$. If we denote by $2 p_{0}\left(2 k T_{0} / m\right)^{-5 / 2}(1+\phi) E$ the velocity distribution function and by $T_{0}\left(1+\Delta \tau+C_{\mathrm{T}} x_{1}\right), p_{0}(1+\Delta P)$, and $\left(2 k T_{0} / m\right)^{1 / 2}\left(u_{\infty}, 0,0\right)$ the temperature, pressure, and flow velocity at a far distance, the problem is formulated as follows:

$$
\begin{aligned}
\zeta_{1} \frac{\partial \phi}{\partial x_{1}} & =\mathscr{L}(\phi), \\
\phi & =\int_{\zeta_{1}^{*}<0} \frac{\left|\zeta_{1}^{*}\right| E\left(\boldsymbol{\zeta}^{*}\right)}{\zeta_{1} E(\boldsymbol{\zeta})} R_{\mathrm{PR}}\left(\boldsymbol{\zeta}^{*}, \zeta\right) \phi^{*} \mathrm{~d} \boldsymbol{\zeta}^{*}, \quad \zeta_{1}>0, x_{1}=0, \\
\phi & \rightarrow h \equiv \Delta P+\left(|\boldsymbol{\zeta}|^{2}-\frac{5}{2}\right) \Delta \tau+\left[\left(|\zeta|^{2}-\frac{5}{2}\right) x_{1}-\zeta_{1} A(|\zeta|)\right] C_{\mathrm{T}}+2 \zeta_{1} u_{\infty}, \quad \text { as } x_{1} \rightarrow \infty,
\end{aligned}
$$

where $R_{\mathrm{PR}}$ is independent of $\boldsymbol{x}$ and $\Delta P, \Delta \tau, C_{\mathrm{T}}$, and $u_{\infty}$ are constants. Note that the heat flow $p_{0}\left(2 k T_{0} / m\right)^{1 / 2}\left(Q_{\infty}, 0,0\right)$ at a far distance is given by

$$
Q_{\infty}=\left\langle\zeta_{1}\left(|\boldsymbol{\zeta}|^{2}-\frac{5}{2}\right) \phi\right\rangle_{x_{1} \rightarrow \infty}=\left\langle\zeta_{1}\left(|\boldsymbol{\zeta}|^{2}-\frac{5}{2}\right) h\right\rangle=-\frac{1}{3}\left\langle|\boldsymbol{\zeta}|^{4} A(|\boldsymbol{\zeta}|)\right\rangle C_{\mathrm{T}}
$$

Denoting by subscript $(1,0)$ the quantities $\phi, h, \Delta P$, and $\Delta \tau$ when $\left(Q_{\infty}, u_{\infty}\right)=(1,0)$ and by subscript $(0,1)$ the counterparts when $\left(Q_{\infty}, u_{\infty}\right)=(0,1)$, we can split the problem as

$$
\begin{aligned}
\phi & =\phi_{(1,0)} Q_{\infty}+\phi_{(0,1)} u_{\infty}, & h & =h_{(1,0)} Q_{\infty}+h_{(0,1)} u_{\infty}, \\
\Delta P & =\Delta P_{(1,0)} Q_{\infty}+\Delta P_{(0,1)} u_{\infty}, & \Delta T & =\Delta T_{(1,0)} Q_{\infty}+\Delta T_{(0,1)} u_{\infty} .
\end{aligned}
$$

As in Sect. 3.1, each of the reduced problems, the case of $\left(Q_{\infty}, u_{\infty}\right)=(1,0)$ or $(0,1)$, is known to have a solution if and only if $\Delta P_{(1,0)}$ and $\Delta \tau_{(1,0)}\left(\right.$ or $\Delta P_{(0,1)}$ and $\left.\Delta \tau_{(0,1)}\right)$ take a special set of values, and the solution $\phi_{(0,1)}\left(\right.$ or $\left.\phi_{(1,0)}\right)$ is unique and approaches $h_{(0,1)}$ (or $h_{(1,0)}$ ) exponentially fast as $x_{1} \rightarrow \infty .{ }^{6}$ With these properties in mind, we make use of Proposition 2 with $\phi^{A}=\phi_{(1,0)}$ and $\phi^{B}=\phi_{(0,1)}$ to have the relation ${ }^{7}$

$$
-\frac{1}{2}\left\langle\zeta_{1} h_{(0,1)}^{-} h_{(1,0)}\right\rangle_{x_{1} \rightarrow \infty}=-\frac{1}{2}\left\langle\zeta_{1} h_{(1,0)}^{-} h_{(0,1)}\right\rangle_{x_{1} \rightarrow \infty}
$$

6 As is seen from the form of the equation and the boundary condition, $\phi_{(0,1)}\left(\right.$ or $\left.\phi_{(1,0)}\right)$ can be sought as a function of $x_{1}, \zeta_{1}$, and $|\boldsymbol{\zeta}|$. This property will be used later in Sect. 3.3.

7 There is a simple analogue in the theory of radiative transfer [18], in which the following two problems in a half space of isotropic scattering field are considered (F. Golse, private communication):

$$
\begin{aligned}
& \mu \partial_{x} f=-f+\langle f\rangle, \quad f(x=0, \mu>0)=\varphi(\mu), \quad f \rightarrow f_{\infty}=\left\langle f_{\infty}\right\rangle \quad \text { as } x \rightarrow \infty, \\
& \mu \partial_{x} g=-g+\langle g\rangle, \quad g(x=0, \mu>0)=0, \quad\langle\mu g\rangle=-1,
\end{aligned}
$$

where $f(x, \mu)$ and $g(x, \mu)(x \geq 0,-1 \leq \mu \leq 1)$, the analogue to $\phi_{(0,1)}$ and $\phi_{(1,0)}$, denote the intensity of radiation, $\langle f\rangle=\frac{1}{2} \int_{-1}^{1} f\left(x, \mu^{\prime}\right) \mathrm{d} \mu^{\prime}$, and $\varphi$ is a given function. Here, concerned is mainly the value of $f_{\infty}$ in the first problem and $g(x=0, \mu<0)$ in the second. We can show that $f_{\infty}=\frac{1}{2} \int_{0}^{1} \varphi\left(\mu^{\prime}\right) g\left(0,-\mu^{\prime}\right) \mathrm{d} \mu^{\prime}$ in a way similar to the derivation of $(10)$, where $g(x=0, \mu>0)=0$ is essential to remove the contribution of $f(x=0, \mu<0)$ from the identity. In this simple analogue, elaborate considerations on the boundary like in Sect. 2 are not necessary. 
which is reduced to

$$
\Delta P_{(1,0)}=\Delta \tau_{(0,1)} .
$$

The relation (18) can be checked by numerical data in the literature. In fact, $\Delta P_{(1,0)}$ and $\Delta \tau_{(0,1)}$ are related to $C_{1}$ and $d_{4}^{*}$ in [10] as $C_{1}=-\frac{1}{3}\left\langle|\zeta|^{4} A(|\zeta|)\right\rangle \Delta P_{(1,0)}$ and $d_{4}^{*}=\Delta \tau_{(0,1)}$, where $C_{1}$ and $d_{4}^{*}$ for the complete condensation condition are given as

$$
\begin{aligned}
& C_{1}=0.55844, \quad d_{4}^{*}=-0.44675 \quad(\text { BGK model }[34,10]), \\
& \left.C_{1}=1.0947, \quad d_{4}^{*}=-0.4557 \quad \text { (Boltzmann equation; hard sphere }[39,37,10]\right) .
\end{aligned}
$$

Note that $\frac{1}{3}\left\langle|\zeta|^{4} A\right\rangle$ is $\frac{5}{2} \gamma_{2}$ in [10] $\left(\gamma_{2}=1\right.$ for the BGK model and $\gamma_{2}=1.922284066$ for the hard-sphere Boltzmann equation). Substituting these values shows the relation (18) to hold.

\subsection{Jump condition for the Stokes set of equations on the condensed phase}

Consider the steady behavior of a slightly rarefied gas around its condensed phase with arbitrary (smooth) shape in the linear regime. According to the asymptotic theory $[11,34$, $12,10]$ for small Knudsen numbers, the overall behavior of the gas can be described fluiddynamically by the Stokes set of equations with a proper set of slip condition for the flow velocity and jump condition for the pressure and temperature. However, a correction is required in a thin layer adjacent to the interface with the thickness of a few mean free paths. The thin layer is called the Knudsen layer, and the correction is correspondingly called the Knudsen-layer correction; the solution of the Stokes set is called the fluid-dynamic part (of the solution of the Boltzmann equation). The study of the Knudsen layer is reduced to several half space problems of the homogeneous or inhomogeneous Boltzmann equation. The problems treated in Sects. 3.1 and 3.2 are typical examples of those reduced problems. By the analyses of the reduced problems, we can obtain the concrete value of the coefficients, the so-called slip and jump coefficients, occurring in the slip and jump conditions. For the details of the asymptotic theory, the reader is referred to $[12,10]$. Here we merely present the general expressions for the pressure and jump conditions derived by the theory. We are going to show some relations among the jump coefficients obtained by the use of the symmetric relation (14).

Let us denote by $\tau$ and $P$ the perturbed temperature and pressure of the fluid-dynamic part and by $\tau_{\mathrm{w}}$ and $P_{\mathrm{w}}$ the perturbed temperature of the condensed phase and the corresponding perturbed saturation pressure of the gas. According to [10], if neglecting the second and higher order effects of the Knudsen number, the difference of $\tau$ and $P$ from $\tau_{\mathrm{w}}$ and $P_{\mathrm{w}}$ at the interface, which is called the temperature jump and pressure jump respectively, is given by

$$
\left[\begin{array}{c}
P-P_{\mathrm{w}} \\
\tau-\tau_{\mathrm{w}}
\end{array}\right]=u_{n}\left[\begin{array}{c}
C_{4}^{*} \\
d_{4}^{*}
\end{array}\right]+\frac{\sqrt{\pi}}{2} \mathrm{Kn}\left\{\frac{\partial \tau}{\partial x_{n}}\left[\begin{array}{c}
C_{1} \\
d_{1}
\end{array}\right]+2 \frac{\partial u_{n}}{\partial x_{n}}\left[\begin{array}{c}
C_{6} \\
d_{6}
\end{array}\right]-2 \bar{\kappa} u_{n}\left[\begin{array}{l}
C_{7} \\
d_{7}
\end{array}\right]\right\}
$$

Here $u_{n}$ is the component inward normal to the interface of the fluid-dynamic part of the dimensionless flow velocity, $x_{n}$ the same component of the dimensionless position vector, and $\bar{\kappa} / L$ is the mean curvature of the interface. ${ }^{8}$ In the above expression, $u_{n}, \partial \tau / \partial x_{n}$, and $\partial u_{n} / \partial x_{n}$ on the right-hand side represent their value at the interface.

As partially mentioned in Sect. 3.2, the coefficients $\left(C_{1}, d_{1}\right)$ and $\left(C_{4}^{*}, d_{4}^{*}\right)$ are related to $\Delta P^{\prime}$ 's and $\Delta \tau$ 's there as $\left(C_{1}, d_{1}\right)=-\frac{5}{2} \gamma_{2}\left(\Delta P_{(1,0)}, \Delta \tau_{(1,0)}\right)$ and $\left(C_{4}^{*}, d_{4}^{*}\right)=\left(\Delta P_{(0,1)}, \Delta \tau_{(0,1)}\right)$.

\footnotetext{
${ }^{8}$ Here and in [10], the curvature is negative when the corresponding center of curvature lies on the gas side.
} 
On the other hand, $\left(C_{6}, d_{6}\right)$ and $\left(C_{7}, d_{7}\right)$ are respectively determined with the solution $\phi_{6}$ and $\phi_{7}$ of the following half space problems:

$$
\begin{aligned}
\zeta_{1} \frac{\partial \phi_{J}}{\partial x_{1}} & =\mathscr{L}\left(\phi_{J}\right)+I_{J}, \quad(J=6,7), \\
\phi_{J} & =\int_{\zeta_{1}^{*}<0} \frac{\left|\zeta_{1}^{*}\right| E\left(\zeta^{*}\right)}{\zeta_{1} E(\zeta)} R_{\mathrm{PR}}\left(\zeta^{*}, \zeta\right) \phi_{J}^{*} \mathrm{~d} \zeta^{*}, \quad \zeta_{1}>0, x_{1}=0, \\
\phi_{J} & \rightarrow h_{J}, \quad \text { as } x_{1} \rightarrow \infty
\end{aligned}
$$

where

$$
\begin{array}{ll}
I_{6}=\frac{1}{2}\left(3 \zeta_{1}^{2}-|\zeta|^{2}\right), & h_{6}=-C_{6}-\left(|\boldsymbol{\zeta}|^{2}-\frac{5}{2}\right) d_{6}+\frac{1}{4}\left(3 \zeta_{1}^{2}-|\zeta|^{2}\right) B(|\zeta|), \\
I_{7}=\frac{1}{2}\left(\zeta_{1}^{2}-|\zeta|^{2}\right) \frac{\partial \psi_{(0,1)}}{\partial \zeta_{1}}, & h_{7}=-C_{7}-\left(|\zeta|^{2}-\frac{5}{2}\right) d_{7},
\end{array}
$$

and $R_{\mathrm{PR}}$ is independent of $\boldsymbol{x}$. The $\psi_{(0,1)}$ is a function of $x_{1}, \zeta_{1}$, and $|\boldsymbol{\zeta}|$ defined by $\psi_{(0,1)}=$ $\phi_{(0,1)}-h_{(0,1)}$ (see the footnote 6) and vanishes exponentially as $x_{1} \rightarrow \infty$.

We first apply Proposition 2 to the pairs of $\phi_{6}$ and $\phi_{(1,0)}$ by putting $\phi^{A}=\phi_{6}$ and $\phi^{B}=$ $\phi_{(1,0)}$ and of $\phi_{6}$ and $\phi_{(0,1)}$ by putting $\phi^{A}=\phi_{6}$ and $\phi^{B}=\phi_{(0,1)}$. Then we eventually obtain

$$
C_{6}=\gamma_{1}-\frac{3}{4} \int_{0}^{\infty}\left(\Omega_{4}^{*}+\Theta_{4}^{*}\right) \mathrm{d} x_{1}, \quad d_{6}=\frac{1}{5 \gamma_{2}}\left(2 \gamma_{3}+3 \int_{0}^{\infty}\left(\Omega_{1}+\Theta_{1}\right) \mathrm{d} x_{1}\right) .
$$

Here we followed the notation in [10], i.e., $\Omega_{4}^{*}+\Theta_{4}^{*}=\frac{2}{3}\left\langle|\zeta|^{2} \psi_{(0,1)}\right\rangle, \Omega_{1}+\Theta_{1}=-\frac{5}{6} \gamma_{2}\left\langle|\zeta|^{2} \psi_{(1,0)}\right\rangle$, where $\gamma_{1} \sim \gamma_{3}$ are those in Sects. 3.1 and 3.2 and $\psi_{(1,0)}=\phi_{(1,0)}-h_{(1,0)}$. The relation (20) can be checked by the numerical data in the literature. In the case of the complete condensation condition, they are given for the BGK model as follows [34,10]:

$$
\begin{aligned}
& C_{6}=0.82085, \quad \int_{0}^{\infty}\left(\Omega_{4}^{*}+\Theta_{4}^{*}\right) \mathrm{d} x_{1}=0.23886, \\
& d_{6}=0.33034, \quad \int_{0}^{\infty}\left(\Omega_{1}+\Theta_{1}\right) \mathrm{d} x_{1}=-0.11609 .
\end{aligned}
$$

Since $\gamma_{1}=\gamma_{2}=\gamma_{3}=1$ for this model, the relation (20) is seen to hold. On the other hand, the values of $C_{6}$ and $d_{6}$ have been unknown for the other cases, and here we report their values for the hard-sphere Boltzmann equation by the use of (20) with the aid of the data of $\psi_{(1,0)}$ and $\psi_{(0,1)}$ available in the literature (e.g., [37,39-41]). By the use of the numerical data in $[40,41]$, the integrals of $\Omega_{4}^{*}+\Theta_{4}^{*}$ and $\Omega_{1}+\Theta_{1}$ are computed as

$$
\int_{0}^{\infty}\left(\Omega_{4}^{*}+\Theta_{4}^{*}\right) \mathrm{d} x_{1}=0.2834, \quad \int_{0}^{\infty}\left(\Omega_{1}+\Theta_{1}\right) \mathrm{d} x_{1}=-0.1943 .
$$

Since $\gamma_{1}=1.270042427, \gamma_{2}=1.922284066$, and $\gamma_{3}=1.947906335$, we have

$$
C_{6}=1.0575, \quad d_{6}=0.3447 \quad \text { (Boltzmann equation; hard-sphere) }
$$

in the case of the complete condensation condition. To our best knowledge, this is the first report on the values of these coefficients for the hard-sphere Boltzmann equation.

Next we apply Proposition 2 to the pairs of $\phi_{7}$ and $\phi_{(1,0)}$ by putting $\phi^{A}=\phi_{7}$ and $\phi^{B}=$ $\phi_{(1,0)}$ and of $\phi_{7}$ and $\phi_{(0,1)}$ by putting $\phi^{A}=\phi_{7}$ and $\phi^{B}=\phi_{(0,1)}$. Then, we eventually obtain

$$
C_{7}=\frac{1}{2} \int_{0}^{\infty}\left\langle\left(\zeta_{1}^{2}-|\zeta|^{2}\right) \psi_{(0,1)}^{-} \frac{\partial \psi_{(0,1)}}{\partial \zeta_{1}}\right\rangle \mathrm{d} x_{1}-\frac{3}{2} \int_{0}^{\infty}\left(\Omega_{4}^{*}+\Theta_{4}^{*}\right) \mathrm{d} x_{1},
$$


$d_{7}=\frac{1}{2} \int_{0}^{\infty}\left\langle\left(\zeta_{1}^{2}-|\zeta|^{2}\right) \psi_{(1,0)}^{-} \frac{\partial \psi_{(0,1)}}{\partial \zeta_{1}}\right\rangle \mathrm{d} x_{1}+\frac{1}{5 \gamma_{2}} \int_{0}^{\infty}\left\langle\left(3 \zeta_{1}^{2}-|\zeta|^{2}\right) A(|\zeta|) \psi_{(0,1)}\right\rangle \mathrm{d} x_{1}$.

The present example demonstrates the advantage of a rather abstract formulation in Sect. 2. The problem for $\phi_{7}$ contains the derivative of non Chapman-Enskog solution $\psi_{(0,1)}$ as the inhomogeneous term, which is typical in the higher-order analyses of the Knudsenlayer. The feature that $I$ (and $h$ ) is not required to be of a specific form is advantageous in such analyses and will allow us to derive further identities for slip and jump coefficients.

\section{Representation theorem on mass, momentum, and heat fluxes}

As noted just before Lemma 2 in Sect. 2.2.2, a remarkable difference of the bounded and three dimensional unbounded domains from the half space is the fact that one may separately discuss the effect of the sources on the boundary. We shall present a fruitful consequence obtained from this property linked to the symmetric relation (14).

\subsection{Problem and formulation}

Consider the steady behavior of a rarefied gas in a domain that is arbitrary except for the conditions to be described later. There is no external force. The state of the gas is so close to the reference equilibrium state at rest with density $\rho_{0}$ and temperature $T_{0}$ that the higher order terms of the deviation from the reference state may be neglected. We shall mainly discuss the general properties of the mass, momentum, and heat transfered to the boundary of the domain under consideration.

Let us denote by $L$ the reference length of the system, by $L \boldsymbol{x}$ the position, by $\left(2 k T_{0} / m\right)^{1 / 2} \boldsymbol{\zeta}$ the molecular velocity, and by $\rho_{0}\left(2 k T_{0} / m\right)^{-3 / 2}(1+\phi) E(\boldsymbol{\zeta})$ the velocity distribution function of gas molecules, where $k$ is the Boltzmann constant and $m$ is the mass of a gas molecule. Then, the behavior of the gas, the perturbed velocity distribution function $\phi$, is described by the following linearized Boltzmann equation:

$$
\zeta_{i} \frac{\partial \phi}{\partial x_{i}}=\frac{2}{\sqrt{\pi}} \frac{1}{\mathrm{Kn}} \mathscr{L}(\phi)
$$

where $\mathrm{Kn}$ is the Knudsen number defined by $\mathrm{Kn}=\ell_{0} / L$ with $\ell_{0}$ being the mean free path of a molecule in the gas at the reference equilibrium state.

Let us denote by $D$ the domain of $\boldsymbol{x}$ representing the gas domain under consideration. The boundary $\partial D$ of the domain is split into two parts: the part $\partial D_{\mathrm{w}}$ representing the real boundary and the part $\partial D_{\mathrm{g}}$ representing the remaining imaginary boundary. As the domain $D$, we consider the following two possibilities:

1. $D$ is a bounded domain.

2. $D$ is an unbounded domain, and the real boundary $\partial D_{\mathrm{w}}$ is confined in a finite region, i.e., there exists a sphere with a finite radius that contains $\partial D_{\mathrm{w}}$ in its interior.

It is important to note that the second assumption excludes unbounded domains in one and two dimensional problems from the subsequent discussions.

As to the real boundary part, we denote by $T_{0}\left(1+\tau_{\mathrm{w}}\right)$ the temperature of the real boundary $\partial D_{w}$, by $p_{0}\left(1+P_{\mathrm{w}}\right)$ the corresponding saturation pressure of the gas, and by 
$\left(2 k T_{0} / m\right)^{1 / 2} \boldsymbol{u}_{\mathrm{w}}$ the velocity of the boundary, where $p_{0}=\left(\rho_{0} / m\right) k T_{0}$. Then, $\phi$ obeys the following condition on $\partial D_{\mathrm{w}}$ :

$$
\phi=g_{\mathrm{w}}+\int_{\zeta_{n}^{*}<0} \frac{\left|\zeta_{n}^{*}\right| E\left(\boldsymbol{\zeta}^{*}\right)}{\left|\zeta_{n}\right| E(\boldsymbol{\zeta})} R\left(\boldsymbol{\zeta}^{*}, \boldsymbol{\zeta} ; \boldsymbol{x}\right)\left(\phi^{*}-g_{\mathrm{w}}^{*}\right) \mathrm{d} \boldsymbol{\zeta}^{*} \quad \text { for } \zeta_{n}>0
$$

Here $R=R_{\mathrm{CR}}$ on the simple boundary and $R=R_{\mathrm{PR}}$ on the interface, where $R_{\mathrm{CR}}$ and $R_{\mathrm{PR}}$ are those in Appendix A that satisfy the condition of detailed balance (31). The function $g_{\mathrm{w}}$ is given by (5). The kernel $R$ multiplied by $\left(2 k T_{0} / m\right)^{3 / 2}$ is the reflection kernel of the real boundary which is at rest with the reference temperature $T_{0}$. In what follows, irrespective of the type of the real boundary (either $R=R_{\mathrm{CR}}$ or $R_{\mathrm{PR}}$ ), we simply write $g_{\mathrm{w}}$ as

$$
g_{\mathrm{w}}=P_{\mathrm{w}}+2 \zeta_{i} u_{\mathrm{w} i}+\left(|\zeta|^{2}-\frac{5}{2}\right) \tau_{\mathrm{w}} \quad \text { on } \partial D_{\mathrm{w}}
$$

because the addition of any $\zeta$-independent term to $g_{\mathrm{w}}$ does not influence the condition (23) because of the third property of $R_{\mathrm{CR}}$ in Appendix A. Note that $u_{\mathrm{w} i} n_{i}=0$ because we are concerned with the steady problem.

On the imaginary boundary $\partial D_{\mathrm{g}}, \phi$ obeys the following condition:

1. When $D$ is bounded, $\partial D_{\mathrm{g}}=\partial D_{\mathrm{g}}^{(\mathrm{i})} \cup \partial D_{\mathrm{g}}^{(\mathrm{iii})}$ and $\phi$ obeys the condition (11a) or (11c):

$$
\begin{aligned}
& \phi(\boldsymbol{x}, \boldsymbol{\zeta})=h_{\mathrm{in}}(\boldsymbol{x}, \boldsymbol{\zeta}) \text { for } \zeta_{n}>0 \text { on } \partial D_{\mathrm{g}}^{(\mathrm{i})}, \\
& \phi(\boldsymbol{x}, \boldsymbol{\zeta})=h(\boldsymbol{x}, \boldsymbol{\zeta})+\int_{\partial D_{\mathrm{g}}^{(\mathrm{iii})}} \int_{\zeta_{n^{\prime}}<0} P\left(\boldsymbol{x}^{\prime}, \boldsymbol{\zeta}^{\prime}, \boldsymbol{x}, \boldsymbol{\zeta}\right)\left(\phi^{\prime}-h^{\prime}\right) \mathrm{d} \boldsymbol{\zeta}^{\prime} \mathrm{d} S^{\prime} \quad \text { for } \zeta_{n}>0 \text { on } \partial D_{\mathrm{g}}^{(\mathrm{iii})}
\end{aligned}
$$

2. When $D$ is unbounded, $\partial D_{\mathrm{g}}=\partial D_{\mathrm{g}}^{(\mathrm{ii})}$, and $\phi$ obeys the condition (11b):

$$
\phi(\boldsymbol{x}, \boldsymbol{\zeta}) \rightarrow h(\boldsymbol{x}, \boldsymbol{\zeta}) \quad \text { as }|\boldsymbol{x}| \rightarrow \infty,
$$

where $h$ is a solution of (22).

Note that $h$ is defined for the whole range of $\zeta$ in each case. As in Sect. 2.2.1, we extend the range of the function $h_{\text {in }}$ from $\zeta_{n}>0$ to the whole range of $\zeta$ and denote the extended function by $h$. The way of extension is arbitrary and does not affect the subsequent results.

Most of the cases, we are concerned with the flow velocity $\left(2 k T_{0} / m\right)^{1 / 2} u_{i}$, heat-flow vector $\frac{1}{2} \rho_{0}\left(2 k T_{0} / m\right)^{3 / 2} Q_{i}$, and stress tensor $p_{0}\left(\delta_{i j}+P_{i j}\right)$ of the gas, where $\delta_{i j}$ is the Kronecker delta. In particular, their component normal to the boundary is of special interest, which will be denoted by $u_{n}, Q_{n}$, and $P_{n j}$ :

$$
u_{n}=u_{i} n_{i}=\left\langle\zeta_{n} \phi\right\rangle, \quad Q_{n}=Q_{i} n_{i}=\left\langle\zeta_{n}\left(|\zeta|^{2}-\frac{5}{2}\right) \phi\right\rangle, \quad P_{n j}=P_{i j} n_{i}=\left\langle 2 \zeta_{n} \zeta_{j} \phi\right\rangle .
$$

Note that they represent the dimensionless inward fluxes of mass, heat, and momentum through the boundary at the position, respectively.

As is noted in Sect. 2.2.2, all the conditions in Proposition 2 are fulfilled by any two solutions of (22), (23), and (25) with $g_{\mathrm{w}}$ given by (24). The first condition is obviously fulfilled by the definition of $R$, while the second is due to Lemma $2 .{ }^{9}$ Thus, the symmetric relation (14) holds between any two solutions discussed in Sect. 4.

\footnotetext{
9 Note that the addition of $P_{\mathrm{w}}$ to $g_{\mathrm{w}}$ made in (24) on a simple boundary does not change the value of the first term of each side of (14) because $\left\langle\zeta_{n} \phi\right\rangle=0$ there.
} 
Table 1 Green functions for elemental sources on $\partial D_{\mathrm{w}}$. The domain may be bounded or unbounded.

\begin{tabular}{clcc}
\hline Green function $^{\mathrm{a}}$ & \multicolumn{2}{c}{ corresponding elemental source $^{\mathrm{b}}$} & note \\
\hline$G^{\left(P ; \boldsymbol{x}_{0}\right)}(\boldsymbol{x}, \boldsymbol{\zeta})$ & $g_{\mathrm{w}}=\boldsymbol{\delta}\left(\boldsymbol{x}-\boldsymbol{x}_{0}\right)$ & $h=0$ & - \\
$G^{\left(T ; \boldsymbol{x}_{0}\right)}(\boldsymbol{x}, \boldsymbol{\zeta})$ & $g_{\mathrm{w}}=\left(|\boldsymbol{\zeta}|^{2}-\frac{5}{2}\right) \boldsymbol{\delta}\left(\boldsymbol{x}-\boldsymbol{x}_{0}\right)$ & $h=0$ & - \\
$G^{\left(\boldsymbol{t} ; \boldsymbol{x}_{0}\right)}(\boldsymbol{x}, \boldsymbol{\zeta})$ & $g_{\mathrm{w}}=2 \zeta_{i} t_{i} \boldsymbol{\delta}\left(\boldsymbol{x}-\boldsymbol{x}_{0}\right)$ & $h=0$ & $G^{\left(\boldsymbol{t} ; \boldsymbol{x}_{0}\right)}(\boldsymbol{x}, \boldsymbol{\zeta})=-G^{\left(-\boldsymbol{t} ; \boldsymbol{x}_{0}\right)}(\boldsymbol{x}, \boldsymbol{\zeta})$ \\
$G^{(P ; \mathbf{S})}(\boldsymbol{x}, \boldsymbol{\zeta})$ & $g_{\mathrm{w}}=\chi_{\mathrm{S}}(\boldsymbol{x})$ & $h=0$ & $G^{(P ; \mathbf{S})}(\boldsymbol{x}, \boldsymbol{\zeta})=\int_{\mathrm{S}} G^{\left(P ; \boldsymbol{x}_{0}\right)}(\boldsymbol{x}, \boldsymbol{\zeta}) \mathrm{dS}_{0}$ \\
$G^{(T ; \mathbf{S})}(\boldsymbol{x}, \boldsymbol{\zeta})$ & $g_{\mathrm{w}}=\left(|\boldsymbol{\zeta}|^{2}-\frac{5}{2}\right) \chi_{\mathrm{S}}(\boldsymbol{x})$ & $h=0$ & $G^{(T ; \mathbf{S})}(\boldsymbol{x}, \boldsymbol{\zeta})=\int_{\mathrm{S}} G^{\left(T ; \boldsymbol{x}_{0}\right)}(\boldsymbol{x}, \boldsymbol{\zeta}) \mathrm{dS}_{0}$ \\
\hline
\end{tabular}

${ }^{\text {a }} \boldsymbol{t}$ is a unit vector tangential to $\partial D_{\mathrm{w}}$ at $\boldsymbol{x}_{0} . \mathrm{S} \subseteq \partial D_{\mathrm{w}}$.

${ }^{\mathrm{b}} \delta$ is the $\delta$-function. $\chi_{\mathrm{S}}(\boldsymbol{x})=1$ for $\boldsymbol{x} \in \mathrm{S}$ and $\chi_{\mathrm{S}}(\boldsymbol{x})=0$ otherwise.

4.2 Preliminary argument - motivation and basic results

If $h=0$ on $\partial D_{\mathrm{g}}$, the perturbation $\phi$ from the reference equilibrium state is induced only by the source $g_{\mathrm{w}}$ on $\partial D_{\mathrm{w}}$. Since $g_{\mathrm{w}}$ is a linear combination of $1, \boldsymbol{\zeta}$ with $\zeta_{n}=0$, and $|\boldsymbol{\zeta}|^{2}-\frac{5}{2}$, we are motivated to consider the response of the system to these elemental sources on $\partial D_{\mathrm{w}}$ and to represent $\phi$ as their superposition.

Let $x_{0}$ be a point on the real boundary $\partial D_{\mathrm{w}}$. We introduce three elemental solutions of the boundary-value problem (22), (23), and (25) listed in the first three lines in Table 1, which we denote by $G^{\left(P ; x_{0}\right)}(\boldsymbol{x}, \boldsymbol{\zeta}), G^{\left(T ; x_{0}\right)}(\boldsymbol{x}, \boldsymbol{\zeta})$, and $G^{\left(t ; x_{0}\right)}(\boldsymbol{x}, \boldsymbol{\zeta})$. Each of them represents the response of the system to the corresponding elemental source put on $\partial D_{\mathrm{w}}$, and thus we call them the Green functions ${ }^{10}$ with respect to $x_{0}$ on $\partial D_{\mathrm{w}}$. If necessary, we call $G^{\left(P ; x_{0}\right)}$, $G^{\left(T ; x_{0}\right)}$, and $G^{\left(t ; x_{0}\right)}$ the Green function for the pressure source, temperature source, and velocity source in the $t$-direction, respectively. Any solution $\phi$ of the boundary-value problem (22), (23), and (25) with $h=0$ can be expressed as a superposition of the Green functions:

$$
\phi=\int_{\partial D_{\mathrm{w}}}\left(P_{\mathrm{w}}\left(\boldsymbol{x}_{0}\right) G^{\left(P ; \boldsymbol{x}_{0}\right)}+\tau_{\mathrm{w}}\left(\boldsymbol{x}_{0}\right) G^{\left(T ; \boldsymbol{x}_{0}\right)}-\left|\boldsymbol{u}_{\mathrm{w}}\left(\boldsymbol{x}_{0}\right)\right| G^{\left(-\hat{\boldsymbol{u}}_{\mathrm{w}} ; \boldsymbol{x}_{0}\right)}\right) \mathrm{d} \mathrm{S}_{0},
$$

where $\hat{\boldsymbol{u}}_{\mathrm{w}}=\boldsymbol{u}_{\mathrm{w}} /\left|\boldsymbol{u}_{\mathrm{w}}\right|$ and $\mathrm{dS}_{0}$ is the surface element at position $\boldsymbol{x}_{0}$. Here and in what follows, we denote the moments of the Green function by putting the corresponding superscript. For instance, $u_{i}^{\left(P ; x_{0}\right)}(\boldsymbol{x})=\left\langle\zeta_{i} G^{\left(P ; x_{0}\right)}(\boldsymbol{x}, \boldsymbol{\zeta})\right\rangle$. The first important observation is a reciprocity of the Green functions in the following sense:

Lemma 3 For any points $\boldsymbol{x}_{0}$ and $\boldsymbol{x}_{1}$ on $\partial D_{\mathrm{w}}$, the following relation holds:

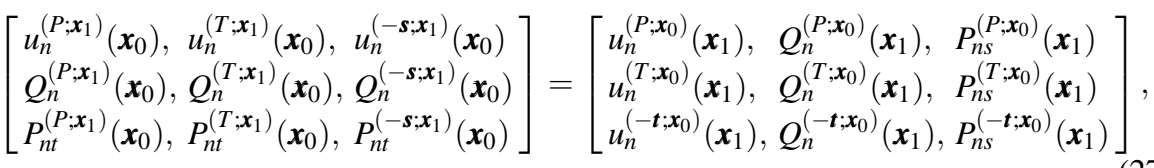

where $P_{n t}=P_{n j} t_{j}, P_{n s}=P_{n j} s_{j}$, and $\boldsymbol{t}$ and $\boldsymbol{s}$ are a unit vector tangential to the boundary at point $\boldsymbol{x}_{0}$ and $\boldsymbol{x}_{1}$ respectively.

Proof Proposition 2 is applicable to any two Green functions (see the last paragraph of Sect. 4.1). With $\phi^{A}=G^{\left(\alpha ; \boldsymbol{x}_{0}\right)}$ and $\phi^{B}=G^{\left(\beta ; \boldsymbol{x}_{1}\right)}(\alpha=P, T,-\boldsymbol{t} ; \beta=P, T,-\boldsymbol{s})$, we apply the symmetric relation (14). Since $I^{A}=0, I^{B}=0, h^{A}=0$, and $h^{B}=0$, (14) is reduced to

$$
\int_{\partial D_{\mathrm{w}}}\left\langle\zeta_{n} g_{\mathrm{w}}^{A-} G^{\left(\beta ; \boldsymbol{x}_{1}\right)}(\boldsymbol{x}, \boldsymbol{\zeta})\right\rangle \mathrm{dS}=\int_{\partial D_{\mathrm{w}}}\left\langle\zeta_{n} g_{\mathrm{w}}^{B-} G^{\left(\alpha ; x_{0}\right)}(\boldsymbol{x}, \boldsymbol{\zeta})\right\rangle \mathrm{dS} .
$$

10 In the present paper, we shall use the term "Green functions" for the solution even when the corresponding elemental source is not a point source. See, for instance, the last two lines in Table 1. 
Let us consider the case $\alpha=P$ and $\beta=T$. Since $g_{\mathrm{w}}^{A}=\delta\left(\boldsymbol{x}-\boldsymbol{x}_{0}\right)$ and $g_{\mathrm{w}}^{B}=\left(|\boldsymbol{\zeta}|^{2}-\frac{5}{2}\right) \delta(\boldsymbol{x}-$ $\left.\boldsymbol{x}_{1}\right)$, performing the surface integration on $\partial D_{\mathrm{w}}$ yields

$$
\left\langle\zeta_{n} G^{\left(T ; x_{1}\right)}\left(x_{0}, \zeta\right)\right\rangle=\left\langle\zeta_{n}\left(|\zeta|^{2}-\frac{5}{2}\right) G^{\left(P ; x_{0}\right)}\left(x_{1}, \zeta\right)\right\rangle,
$$

which is no other than the equality $u_{n}^{\left(T ; \boldsymbol{x}_{1}\right)}\left(\boldsymbol{x}_{0}\right)=Q_{n}^{\left(P ; \boldsymbol{x}_{0}\right)}\left(\boldsymbol{x}_{1}\right)$. Other equalities can be proved in the same way by changing the pair of $\alpha$ and $\beta$.

Lemma 3 leads to the following important consequence, if it is linked to (26):

Corollary 1 Consider the boundary-value problem (22), (23), and (25) with $h=0$. For any point $x_{0}$ on $\partial D_{\mathrm{w}}$, the fluxes normal to the boundary can be expressed by

$$
\left[\begin{array}{c}
u_{n}\left(\boldsymbol{x}_{0}\right) \\
Q_{n}\left(\boldsymbol{x}_{0}\right) \\
P_{n t}\left(\boldsymbol{x}_{0}\right)
\end{array}\right]=\int_{\partial D_{\mathrm{w}}}\left[\begin{array}{c}
P_{\mathrm{w}}(\boldsymbol{x}) u_{n}^{\left(P ; \boldsymbol{x}_{0}\right)}(\boldsymbol{x})+\tau_{\mathrm{w}}(\boldsymbol{x}) Q_{n}^{\left(P ; \boldsymbol{x}_{0}\right)}(\boldsymbol{x})-u_{\mathrm{w} j}(\boldsymbol{x}) P_{n j}^{\left(P ; \boldsymbol{x}_{0}\right)}(\boldsymbol{x}) \\
P_{\mathrm{w}}(\boldsymbol{x}) u_{n}^{\left(T ; \boldsymbol{x}_{0}\right)}(\boldsymbol{x})+\tau_{\mathrm{w}}(\boldsymbol{x}) Q_{n}^{\left(T ; \boldsymbol{x}_{0}\right)}(\boldsymbol{x})-u_{\mathrm{w} j}(\boldsymbol{x}) P_{n j}^{\left(T ; \boldsymbol{x}_{0}\right)}(\boldsymbol{x}) \\
P_{\mathrm{w}}(\boldsymbol{x}) u_{n}^{\left(-t ; \boldsymbol{x}_{0}\right)}(\boldsymbol{x})+\tau_{\mathrm{w}}(\boldsymbol{x}) Q_{n}^{\left(-\boldsymbol{t} ; \boldsymbol{x}_{0}\right)}(\boldsymbol{x})-u_{\mathrm{w} j}(\boldsymbol{x}) P_{n j}^{\left(-\boldsymbol{t} ; \boldsymbol{x}_{0}\right)}(\boldsymbol{x})
\end{array}\right] \mathrm{dS},
$$

where $\boldsymbol{t}$ is a unit vector tangential to the boundary at $\boldsymbol{x}_{0}$.

Proof The superposition (26) yields

$$
\left[\begin{array}{c}
u_{n}\left(\boldsymbol{x}_{0}\right) \\
Q_{n}\left(\boldsymbol{x}_{0}\right) \\
P_{n t}\left(\boldsymbol{x}_{0}\right)
\end{array}\right]=\int_{\partial D_{\mathrm{w}}}\left[\begin{array}{c}
P_{\mathrm{w}}(\boldsymbol{x}) u_{n}^{(P ; \boldsymbol{x})}\left(\boldsymbol{x}_{0}\right)+\tau_{\mathrm{w}}(\boldsymbol{x}) u_{n}^{(T ; \boldsymbol{x})}\left(\boldsymbol{x}_{0}\right)-\left|\boldsymbol{u}_{\mathrm{w}}(\boldsymbol{x})\right| u_{n}^{\left(-\hat{\boldsymbol{u}}_{w} ; \boldsymbol{x}\right)}\left(\boldsymbol{x}_{0}\right) \\
P_{\mathrm{w}}(\boldsymbol{x}) Q_{n}^{(P ; \boldsymbol{x})}\left(\boldsymbol{x}_{0}\right)+\tau_{\mathrm{w}}(\boldsymbol{x}) Q_{n}^{(T ; \boldsymbol{x})}\left(\boldsymbol{x}_{0}\right)-\left|\boldsymbol{u}_{\mathrm{w}}(\boldsymbol{x})\right| Q_{n}^{\left(-\hat{\boldsymbol{u}}_{w} ; \boldsymbol{x}\right)}\left(\boldsymbol{x}_{0}\right) \\
P_{\mathrm{w}}(\boldsymbol{x}) P_{n t}^{(P ; \boldsymbol{x})}\left(\boldsymbol{x}_{0}\right)+\tau_{\mathrm{w}}(\boldsymbol{x}) P_{n t}^{(T ; \boldsymbol{x})}\left(\boldsymbol{x}_{0}\right)-\left|\boldsymbol{u}_{\mathrm{w}}(\boldsymbol{x})\right| P_{n t}^{\left(-\hat{\boldsymbol{u}}_{w} ; \boldsymbol{x}\right)}\left(\boldsymbol{x}_{0}\right)
\end{array}\right] \mathrm{dS},
$$

and the substitution of (27) leads to the desired expression.

Corollary 1 shows that each of the mass, tangential momentum, and heat fluxes at a point $\boldsymbol{x}_{0}$ on the real boundary $\partial D_{\mathrm{w}}$ is expressed as a weighted sum of the fluxes over the boundary $\partial D_{\mathrm{w}}$ induced by the corresponding Green function with respect to that point. The correspondence is as follows: the mass flux $\Leftrightarrow$ the pressure source, the heat flux $\Leftrightarrow$ the temperature source, the momentum flux $\Leftrightarrow$ the velocity source.

\subsection{Representation theorem}

Corollary 1 implies a reduction of the original problem to a single elemental problem of finding the Green function corresponding to the flux of interest. A natural question arises whether a similar representation could be obtained for general situations such that $h$ is not necessarily zero. Fortunately, we can give an affirmative answer to this question, which we present here. The key to the generalization is the fact that one can recover Corollary 1 by directly applying Proposition 2 to the pair of $\phi$ in Sect. 4.2 and the Green function. To be specific, the representations of $u_{n}\left(\boldsymbol{x}_{0}\right), Q_{n}\left(\boldsymbol{x}_{0}\right), P_{n t}\left(\boldsymbol{x}_{0}\right)$ are respectively recovered by the application of Proposition 2 to the pairs of $\phi$ and $G^{\left(P ; x_{0}\right)}, \phi$ and $G^{\left(T ; x_{0}\right)}$, and $\phi$ and $G^{\left(-t ; x_{0}\right)}$. This alternative approach enables us to perform the generalization by the use of suitable elemental sources and the corresponding Green functions. We call the set of expressions thus obtained the representation theorem on mass, momentum, and heat fluxes.

Actually, there are five versions of the representation theorem depending on the situation. We present them in Sects. 4.3.1-4.3.3. 


\subsubsection{Representation of fluxes through $\partial D_{\mathrm{w}}$}

Proposition 3 (Representation theorem 1) Consider the boundary-value problem (22), (23), and (25). The fluxes of mass, heat, and tangential momentum through $\partial D_{\mathrm{w}}$ at $\boldsymbol{x}_{0}$ are represented in terms of the corresponding Green function, irrespective of whether $D$ is bounded or unbounded:

$$
\left[\begin{array}{c}
u_{n}\left(\boldsymbol{x}_{0}\right) \\
Q_{n}\left(\boldsymbol{x}_{0}\right) \\
P_{n t}\left(\boldsymbol{x}_{0}\right)
\end{array}\right]=\int_{\partial D_{\mathrm{w}}}\left[\begin{array}{c}
P_{\mathrm{w}} u_{n}^{\left(P ; \boldsymbol{x}_{0}\right)}+\tau_{\mathrm{w}} Q_{n}^{\left(P ; \boldsymbol{x}_{0}\right)}-u_{\mathrm{w} i} P_{n i}^{\left(P ; \boldsymbol{x}_{0}\right)} \\
P_{\mathrm{w}} u_{n}^{\left(T ; \boldsymbol{x}_{0}\right)}+\tau_{\mathrm{w}} Q_{n}^{\left(T ; \boldsymbol{x}_{0}\right)}-u_{\mathrm{w} i} P_{n i}^{\left(T ; \boldsymbol{x}_{0}\right)} \\
P_{\mathrm{w}} u_{n}^{\left(-t ; \boldsymbol{x}_{0}\right)}+\tau_{\mathrm{w}} Q_{n}^{\left(-\boldsymbol{t} ; \boldsymbol{x}_{0}\right)}-u_{\mathrm{w} i} P_{n i}^{\left(-\boldsymbol{t} ; \boldsymbol{x}_{0}\right)}
\end{array}\right] \mathrm{dS}+\int_{\partial D_{\mathrm{g}}}\left[\begin{array}{c}
\left\langle\zeta_{n} h^{-} G^{\left(P ; \boldsymbol{x}_{0}\right)}\right\rangle \\
\left\langle\zeta_{n} h^{-} G^{\left(T ; x_{0}\right)}\right\rangle \\
\left\langle\zeta_{n} h^{-} G^{\left(-\boldsymbol{t} ; \boldsymbol{x}_{0}\right)}\right\rangle
\end{array}\right] \mathrm{dS},
$$

where $\boldsymbol{t}$ is an arbitrary unit vector tangential to the boundary at $\boldsymbol{x}_{0} \cdot{ }^{11}$ The way of extension of $h_{\mathrm{in}}$ on $\partial D_{\mathrm{g}}^{(\mathrm{i})}$ does not influence the relation. ${ }^{12}$

Proof We apply Proposition 2 to the pair of $\phi^{B}=G^{\left(\alpha ; x_{0}\right)}(\alpha=P, T,-t)$ and $\phi^{A}=\phi$, where $\phi$ is the solution of the boundary-value problem (22), (23), and (25). Since $I^{A}=0, g_{\mathrm{w}}^{A}=g_{\mathrm{w}}$, $h^{A}=h, I^{B}=0$, and $h^{B}=0$, the symmetric relation (14) is reduced to

$$
\int_{\partial D_{\mathrm{w}}}\left\langle\zeta_{n} g_{\mathrm{w}}^{B-} \phi\right\rangle \mathrm{d} \mathrm{S}=\int_{\partial D_{\mathrm{w}}}\left\langle\zeta_{n} g_{\mathrm{w}}^{-} G^{\left(\alpha ; x_{0}\right)}\right\rangle \mathrm{dS}+\int_{\partial D_{\mathrm{g}}}\left\langle\zeta_{n} h^{-} G^{\left(\alpha ; x_{0}\right)}\right\rangle \mathrm{dS},
$$

where $g_{\mathrm{w}}^{B}=\delta\left(\boldsymbol{x}-\boldsymbol{x}_{0}\right),\left(|\boldsymbol{\zeta}|^{2}-\frac{5}{2}\right) \delta\left(\boldsymbol{x}-\boldsymbol{x}_{0}\right)$, and $-2 \zeta_{i} t_{i} \delta\left(\boldsymbol{x}-\boldsymbol{x}_{0}\right)$ for $\alpha=P, T$, and $\boldsymbol{t}$, respectively. Substitution of the specific form of $g_{\mathrm{w}}^{B}$ and $g_{\mathrm{w}}$ yields the desired representation.

Proposition 3 can be transformed into the statement on the fluxes through an arbitrary area on $\partial D_{\mathrm{w}}$, which is useful in many applications. To derive it, we introduce the Green functions with respect to an area on $\partial D_{\mathrm{w}}$ defined in the last two lines in Table 1. If we denote by $\rho_{0} L^{2}\left(2 k T_{0} / m\right)^{1 / 2} \mathscr{M}\left(A_{\mathrm{w}}\right)$ and $\frac{1}{2} \rho_{0} L^{2}\left(2 k T_{0} / m\right)^{3 / 2} \mathscr{Q}\left(A_{\mathrm{w}}\right)$ the mass and heat transfered to an area $A_{\mathrm{w}}$ on $\partial D_{\mathrm{w}}$ per unit time, they are written in terms of $u_{n}$ and $Q_{n}$ as

$$
\left[\begin{array}{c}
\mathscr{M}\left(A_{\mathrm{w}}\right) \\
\mathscr{Q}\left(A_{\mathrm{w}}\right)
\end{array}\right]=-\int_{A_{\mathrm{w}}}\left[\begin{array}{l}
u_{n}\left(\boldsymbol{x}_{0}\right) \\
Q_{n}\left(\boldsymbol{x}_{0}\right)
\end{array}\right] \mathrm{dS}_{0} .
$$

Substituting the representation in Proposition 3 and performing the surface integration with respect to $x_{0}$ leads to the representation in terms of the Green functions with respect to $A_{\mathrm{w}}:{ }^{13}$

Proposition 4 (Representation theorem 2) Consider the boundary-value problem (22), (23), and (25). Irrespective of whether D is bounded or unbounded, the outward fluxes of mass and heat through an area $A_{\mathrm{w}}$ on $\partial D_{\mathrm{w}}$ are represented in terms of the corresponding Green function with respect to this area:

$$
\left[\begin{array}{c}
\mathscr{M}\left(A_{\mathrm{w}}\right) \\
\mathscr{Q}\left(A_{\mathrm{w}}\right)
\end{array}\right]=-\int_{\partial D_{\mathrm{w}}}\left[\begin{array}{c}
P_{\mathrm{w}} u_{n}^{\left(P ; A_{\mathrm{w}}\right)}+\tau_{\mathrm{w}} Q_{n}^{\left(P ; A_{\mathrm{w}}\right)}-u_{\mathrm{w} i} P_{n i}^{\left(P ; A_{\mathrm{w}}\right)} \\
P_{\mathrm{w}} u_{n}^{\left(T ; A_{\mathrm{w}}\right)}+\tau_{\mathrm{w}} Q_{n}^{\left(T ; A_{\mathrm{w}}\right)}-u_{\mathrm{w} i} P_{n i}^{\left(T ; A_{\mathrm{w}}\right)}
\end{array}\right] \mathrm{dS}-\int_{\partial D_{\mathrm{g}}}\left[\begin{array}{c}
\left\langle\zeta_{n} h^{-} G^{\left(P ; A_{\mathrm{w}}\right)}\right\rangle \\
\left\langle\zeta_{n} h^{-} G^{\left(T ; A_{\mathrm{w}}\right)}\right\rangle
\end{array}\right] \mathrm{dS} .
$$

11 We may consider the Green function $G^{\left(\boldsymbol{n} \boldsymbol{x}_{0}\right)}$ that is the solution of the problem (22), (23), and (25) with $h=0$ and $g_{\mathrm{w}}=2 \zeta_{n} \boldsymbol{\delta}\left(\boldsymbol{x}-\boldsymbol{x}_{0}\right)$. Then, we can derive the representation for an arbitrary component of the force. In the present work, however, we restrict ourselves to derive a representation in terms of the Green functions satisfying the physical requirement $\boldsymbol{u}_{\mathrm{w}} \cdot \boldsymbol{n}=0$. The same is true for the general representations of the torque on the real boundary.

12 On $\partial D_{\mathrm{g}}^{(\mathrm{i})}, h$ is an arbitrary extension of $h_{\text {in }}$ from $\zeta_{n}>0$ to the whole range of $\zeta$, and hence $h^{-}$for $\zeta_{n}>0$ is the extended part. The arbitrariness of this part is killed in the moment $\left\langle\zeta_{n} h^{-} G^{\left(\alpha ; x_{0}\right)}\right\rangle(\alpha=P, T,-\boldsymbol{t})$, because $G^{\left(\alpha ; x_{0}\right)}=0$ for $\zeta_{n}>0$ by definition.

13 The same representation is obtained by applying Proposition 2 to the pair of $\phi^{B}=G^{\left(\alpha ; A_{\mathrm{w}}\right)}$ and $\phi^{A}=\phi$ directly, which would be a less demanding way in mathematical rigor. 
Table 2 Green functions for elemental sources on $\partial D_{\mathrm{g}}$ for a bounded domain

\begin{tabular}{cllc}
\hline Green function $^{\mathrm{a}}$ & \multicolumn{2}{c}{ corresponding elemental source $^{\mathrm{b}}$} & note \\
\hline$G^{\left(P ; \boldsymbol{x}_{0}\right)}(\boldsymbol{x}, \boldsymbol{\zeta})$ & $g_{\mathrm{w}}=0$ & $h=\delta\left(\boldsymbol{x}-\boldsymbol{x}_{0}\right)$ & - \\
$G^{\left(T ; \boldsymbol{x}_{0}\right)}(\boldsymbol{x}, \boldsymbol{\zeta})$ & $g_{\mathrm{w}}=0$ & $h=\left(|\boldsymbol{\zeta}|^{2}-\frac{5}{2}\right) \boldsymbol{\delta}\left(\boldsymbol{x}-\boldsymbol{x}_{0}\right)$ & - \\
$G^{\left(\ell ; \boldsymbol{x}_{0}\right)}(\boldsymbol{x}, \boldsymbol{\zeta})$ & $g_{\mathrm{w}}=0$ & $h=2 \zeta_{i} \ell_{i} \boldsymbol{\delta}\left(\boldsymbol{x}-\boldsymbol{x}_{0}\right)$ & $G^{\left(\ell ; x_{0}\right)}(\boldsymbol{x}, \boldsymbol{\zeta})=-G^{\left(-\ell ; \boldsymbol{x}_{0}\right)}(\boldsymbol{x}, \boldsymbol{\zeta})$ \\
$G^{(P ; \mathbf{S})}(\boldsymbol{x}, \boldsymbol{\zeta})$ & $g_{\mathrm{w}}=0$ & $h=\chi_{\mathrm{S}}(\boldsymbol{x})$ & $G^{(P ; \mathrm{S})}(\boldsymbol{x}, \boldsymbol{\zeta})=\int_{\mathrm{S}} G^{\left(P ; x_{0}\right)}(\boldsymbol{x}, \boldsymbol{\zeta}) \mathrm{dS}_{0}$ \\
$G^{(T ; \mathrm{S})}(\boldsymbol{x}, \boldsymbol{\zeta})$ & $g_{\mathrm{w}}=0$ & $h=\left(|\boldsymbol{\zeta}|^{2}-\frac{5}{2}\right) \chi_{\mathrm{S}}(\boldsymbol{x})$ & $G^{(T ; \mathbf{S})}(\boldsymbol{x}, \boldsymbol{\zeta})=\int_{\mathrm{S}} G^{\left(T ; \boldsymbol{x}_{0}\right)}(\boldsymbol{x}, \boldsymbol{\zeta}) \mathrm{dS}_{0}$ \\
$G^{(\ell ; \mathrm{S})}(\boldsymbol{x}, \boldsymbol{\zeta})$ & $g_{\mathrm{w}}=0$ & $h=2 \zeta_{i} \ell_{i} \chi_{\mathrm{S}}(\boldsymbol{x})$ & $G^{(\boldsymbol{\ell} ; \mathrm{S})}(\boldsymbol{x}, \boldsymbol{\zeta})=\int_{\mathrm{S}} G^{\left(\ell ; x_{0}\right)}(\boldsymbol{x}, \boldsymbol{\zeta}) \mathrm{dS}_{0}$ \\
& & & $G^{(\ell, \mathrm{S})}(\boldsymbol{x}, \boldsymbol{\zeta})=-G^{(-\ell ; \mathrm{S})}(\boldsymbol{x}, \boldsymbol{\zeta})$
\end{tabular}

${ }^{\mathrm{a}} \ell$ is an arbitrary unit vector. $\mathrm{S} \subseteq \partial D_{\mathrm{g}}$.

b See the footnote $\mathrm{b}$ in Table 1.

The way of extension of $h_{\mathrm{in}}$ on $\partial D_{\mathrm{g}}^{(\mathrm{i})}$ does not influence the relation (see footnote 12). ${ }^{14}$

\subsubsection{Representation of fluxes through $\partial D_{\mathrm{g}}$ for a bounded domain}

As to the fluxes through $\partial D_{\mathrm{g}}$, we need to discuss the bounded and unbounded domains separately. Here, we focus on the former and introduce the Green functions listed in Table 2. The main difference from the previous case lies in the Green function for the velocity source, i.e., the direction of the velocity source $\ell$ is not necessarily tangential to the boundary but rather arbitrary. This feature allows us to have the representation of not only the mass and heat fluxes but also the momentum flux through an arbitrary area on $\partial D_{\mathrm{g}}$. We first present the representation for fluxes through a point on $\partial D_{\mathrm{g}}$. The proof is similar to that in Sect. 4.3.1 and is omitted for the sake of brevity.

Proposition 5 (Representation theorem 3) Consider the boundary-value problem (22), (23), and (25a) or (25b) for a bounded domain. The fluxes of mass, heat, and tangential momentum through $\partial D_{\mathrm{g}}$ at $\boldsymbol{x}_{\mathrm{g}}$ are represented in terms of the corresponding Green function:

$$
\begin{array}{r}
{\left[\begin{array}{c}
u_{n}\left(\boldsymbol{x}_{\mathrm{g}}\right) \\
Q_{n}\left(\boldsymbol{x}_{\mathrm{g}}\right) \\
P_{n \ell}\left(\boldsymbol{x}_{\mathrm{g}}\right)
\end{array}\right]=\int_{\partial D_{\mathrm{w}}}\left[\begin{array}{c}
P_{\mathrm{w}} u_{n}^{\left(P ; \boldsymbol{x}_{\mathrm{g}}\right)}+\tau_{\mathrm{w}} Q_{n}^{\left(P ; \boldsymbol{x}_{\mathrm{g}}\right)}-u_{\mathrm{w} j} P_{n j}^{\left(P ; \boldsymbol{x}_{\mathrm{g}}\right)} \\
P_{\mathrm{w}} u_{n}^{\left(T ; \boldsymbol{x}_{\mathrm{g}}\right)}+\tau_{\mathrm{w}} Q_{n}^{\left(T ; \boldsymbol{x}_{\mathrm{g}}\right)}-u_{\mathrm{w} j} P_{n j}^{\left(T ; \boldsymbol{x}_{\mathrm{g}}\right)} \\
P_{\mathrm{w}} u_{n}^{\left(-\ell ; \boldsymbol{x}_{\mathrm{g}}\right)}+\tau_{\mathrm{w}} Q_{n}^{\left(-\ell ; \boldsymbol{x}_{\mathrm{g}}\right)}-u_{\mathrm{w} j} P_{n j}^{\left(-\ell ; \boldsymbol{x}_{\mathrm{g}}\right)}
\end{array}\right] \mathrm{dS}} \\
+\int_{\partial D_{\mathrm{g}}}\left[\begin{array}{c}
\left\langle\zeta_{n} h^{-} G^{\left(P ; x_{\mathrm{g}}\right)}\right\rangle \\
\left\langle\zeta_{n} h^{-} G^{\left(T ; \boldsymbol{x}_{\mathrm{g}}\right)}\right\rangle \\
\left\langle\zeta_{n} h^{-} G^{\left(-\ell ; x_{\mathrm{g}}\right)}\right\rangle
\end{array}\right] \mathrm{dS}+\left[\begin{array}{c}
\left\langle\zeta_{n} h\left(\boldsymbol{x}_{\mathrm{g}}, \boldsymbol{\zeta}\right)\right\rangle \\
\left\langle\zeta_{n}\left(|\boldsymbol{\zeta}|^{2}-\frac{5}{2}\right) h\left(\boldsymbol{x}_{\mathrm{g}}, \boldsymbol{\zeta}\right)\right\rangle \\
\left\langle 2 \zeta_{n} \zeta_{j} \ell_{j} h\left(\boldsymbol{x}_{\mathrm{g}}, \boldsymbol{\zeta}\right)\right\rangle
\end{array}\right]
\end{array}
$$

where $P_{n \ell}=P_{n j} \ell_{j}$ and $\ell$ is an arbitrary unit vector. The way of extension of $h_{\mathrm{in}}$ on $\partial D_{\mathrm{g}}^{(\mathrm{i})}$ does not influence the relation. ${ }^{15}$

Next, we show the representation of the fluxes through an area on $\partial D_{\mathrm{g}}$. Let us denote by $\rho_{0} L^{2}\left(2 k T_{0} / m\right)^{1 / 2} \mathscr{M}\left(A_{\mathrm{g}}\right), \frac{1}{2} \rho_{0} L^{2}\left(2 k T_{0} / m\right)^{3 / 2} \mathscr{Q}\left(A_{\mathrm{g}}\right)$, and $p_{0} L^{2} \mathscr{F}_{\ell}\left(A_{\mathrm{g}}\right)$ the mass, heat, and

\footnotetext{
14 We exclude from the general discussion the possibility of the Green function for the velocity source, because, in general, the direction of the vector $\boldsymbol{t}$ tangential to the boundary depends on the position.

15 The reason is slightly different from the case explained in footnote 12 . The extended part of $h^{-}$is for $\zeta_{n}>0$. By definition, $G^{\left(\alpha ; x_{\mathrm{g}}\right)}=\delta\left(\boldsymbol{x}-\boldsymbol{x}_{\mathrm{g}}\right),\left(|\boldsymbol{\zeta}|^{2}-\frac{5}{2}\right) \delta\left(\boldsymbol{x}-\boldsymbol{x}_{\mathrm{g}}\right)$, or $-2 \zeta_{j} \ell_{j} \delta\left(\boldsymbol{x}-\boldsymbol{x}_{\mathrm{g}}\right)(\alpha=P, T$, or $-\boldsymbol{\ell})$ in this range, so that the contributions of the extended part to the second and third terms on the right-hand side cancel out each other.
} 
Table 3 Green functions for elemental sources at infinity for unbounded domain

\begin{tabular}{|c|c|c|c|}
\hline Green function ${ }^{\mathrm{a}}$ & correspc & nding elemental source & note \\
\hline$G^{(P ; \infty)}(\boldsymbol{x}, \boldsymbol{\zeta})$ & $g_{\mathrm{w}}=0$ & $h=1$ & - \\
\hline$G^{(T ; \infty)}(\boldsymbol{x}, \boldsymbol{\zeta})$ & $g_{\mathrm{w}}=0$ & $h=\left(|\boldsymbol{\zeta}|^{2}-\frac{5}{2}\right)$ & - \\
\hline$G^{(\ell ; \infty)}(\boldsymbol{x}, \boldsymbol{\zeta})$ & $g_{\mathrm{w}}=0$ & $h=2 \zeta_{i} \ell_{i}$ & $G^{(\ell ; \infty)}(\boldsymbol{x}, \boldsymbol{\zeta})=-G^{(-\ell ; \infty)}(\boldsymbol{x}, \boldsymbol{\zeta})$ \\
\hline$G^{(\underline{\Omega}(\ell) ; \infty)}(\boldsymbol{x}, \boldsymbol{\zeta})$ & $g_{\mathrm{w}}=0$ & $h=2 \varepsilon_{i j k} \zeta_{i} \ell_{j} x_{k}$ & $G^{(\underline{\Omega}(\ell) ; \infty)}(\boldsymbol{x}, \boldsymbol{\zeta})=-G^{(-\underline{\Omega}(\ell) ; \infty)}(\boldsymbol{x}, \boldsymbol{\zeta})$ \\
\hline
\end{tabular}

momentum in the $\boldsymbol{\ell}$-direction transfered to the area $A_{\mathrm{g}}$ on $\partial D_{\mathrm{g}}$ for a bounded domain per unit time. $p_{0} L^{2} \mathscr{F}_{\ell}\left(A_{\mathrm{g}}\right)$ may be regarded as the force acting on the area $A_{\mathrm{g}}$ in the $\ell$-direction. They are written in terms of $u_{n}, Q_{n}$ and $P_{n \ell}$ as

$$
\left[\begin{array}{c}
\mathscr{M}\left(A_{\mathrm{g}}\right) \\
\mathscr{Q}\left(A_{\mathrm{g}}\right) \\
\mathscr{F}_{\ell}\left(A_{\mathrm{g}}\right)
\end{array}\right]=-\int_{A_{\mathrm{g}}}\left[\begin{array}{c}
u_{n}(\boldsymbol{x}) \\
Q_{n}(\boldsymbol{x}) \\
P_{n \ell}(\boldsymbol{x})
\end{array}\right] \mathrm{dS} .
$$

Again, we just show the result and omit the proof similar to that in Sect. 4.3.1.

Proposition 6 (Representation theorem 4) Consider the boundary-value problem (22), (23), and (25a) or (25b) for a bounded domain. The outward fluxes of mass, heat, and momentum through an arbitrary area $A_{\mathrm{g}}$ on $\partial D_{\mathrm{g}}$ are represented in terms of the corresponding Green function with respect to this area:

$$
\begin{aligned}
& {\left[\begin{array}{c}
\mathscr{M}\left(A_{\mathrm{g}}\right) \\
\mathscr{Q}\left(A_{\mathrm{g}}\right) \\
\mathscr{F}_{\ell}\left(A_{\mathrm{g}}\right)
\end{array}\right]=-\int_{\partial D_{\mathrm{w}}}\left[\begin{array}{c}
P_{\mathrm{w}} u_{n}^{\left(P ; A_{\mathrm{g}}\right)}+\tau_{\mathrm{w}} Q_{n}^{\left(P ; A_{\mathrm{g}}\right)}-u_{\mathrm{w} j} P_{n j}^{\left(P ; A_{\mathrm{g}}\right)} \\
P_{\mathrm{w}} u_{n}^{\left(T ; A_{\mathrm{g}}\right)}+\tau_{\mathrm{w}} Q_{n}^{\left(T ; A_{\mathrm{g}}\right)}-u_{\mathrm{w} j} P_{n j}^{\left(T ; A_{\mathrm{g}}\right)} \\
P_{\mathrm{w}} u_{n}^{\left(-\ell ; A_{\mathrm{g}}\right)}+\tau_{\mathrm{w}} Q_{n}^{\left(-\ell ; A_{\mathrm{g}}\right)}-u_{\mathrm{w} j} P_{n j}^{\left(-\ell ; A_{\mathrm{g}}\right)}
\end{array}\right] \mathrm{dS}} \\
& -\int_{\partial D_{\mathrm{g}}}\left[\begin{array}{c}
\left\langle\zeta_{n} h^{-} G^{\left(P ; A_{\mathrm{g}}\right)}\right\rangle \\
\left\langle\zeta_{n} h^{-} G^{\left(T ; A_{\mathrm{g}}\right)}\right\rangle \\
\left\langle\zeta_{n} h^{-} G^{\left(-\ell ; A_{\mathrm{g}}\right)}\right\rangle
\end{array}\right] \mathrm{dS}-\int_{A_{\mathrm{g}}}\left[\begin{array}{c}
\left\langle\zeta_{n} h\right\rangle \\
\left\langle\zeta_{n}\left(|\zeta|^{2}-\frac{5}{2}\right) h\right\rangle \\
\left\langle 2 \zeta_{n} \zeta_{j} \ell, h\right\rangle
\end{array}\right] \mathrm{dS},
\end{aligned}
$$

where $\ell$ is an arbitrary unit vector. The way of extension of $h_{\text {in }}$ on $\partial D_{\mathrm{g}}^{(\mathrm{i})}$ does not influence the relation (see footnote 15 ).

\subsubsection{Representation of fluxes through $\partial D_{\mathrm{g}}$ for an unbounded domain}

We finally turn to the representation of the fluxes passing through $\partial D_{\mathrm{g}}$ for an unbounded domain and introduce the Green functions listed in Table 3. The main difference from the previous cases is the fact that they are the Green functions for the sources on the whole area of $\partial D_{\mathrm{g}}$ and that a new type of elemental source, which we shall call the rotation source around $\ell$-axis, is introduced in the last line of the table. It should be reminded that in the present case $h$ is required to satisfy (22) and that any linearized local Maxwellian satisfying (22) is limited to a linear combination of the elemental sources in Table 3.

Let us denote by $\rho_{0} L^{2}\left(2 k T_{0} / m\right)^{1 / 2} \mathscr{M}(\infty), \frac{1}{2} \rho_{0} L^{2}\left(2 k T_{0} / m\right)^{3 / 2} \mathscr{Q}(\infty), p_{0} L^{2} \mathscr{F}_{\ell}(\infty)$, and $p_{0} L^{3} \mathscr{T}_{\ell}(\infty)$ the mass, heat, momentum in the $\ell$-direction, and angular momentum around the $\ell$-axis transfered to a far field (or $\partial D_{\mathrm{g}}$ ) per unit time. $p_{0} L^{2} \mathscr{F}_{\ell}(\infty)$ and $p_{0} L^{3} \mathscr{T}_{\ell}\left(D_{\mathrm{g}}^{\infty}\right)$ 
may be regarded as the force and the torque (or the moment of force) acting on $\partial D_{\mathrm{g}}$ in the $\ell$-direction. They are written in terms of $u_{n}, Q_{n}$ and $P_{n j}$ as

$$
\left[\begin{array}{c}
\mathscr{M}(\infty) \\
\mathscr{Q}(\infty) \\
\mathscr{F}_{\ell}(\infty) \\
\mathscr{T}_{\ell}(\infty)
\end{array}\right]=-\int_{\partial D_{\mathrm{g}}}\left[\begin{array}{c}
u_{n}(\boldsymbol{x}) \\
Q_{n}(\boldsymbol{x}) \\
P_{n \ell}(\boldsymbol{x}) \\
\ell_{i} \varepsilon_{i j k} x_{j} P_{n k}(\boldsymbol{x})
\end{array}\right] \mathrm{dS} .
$$

Applying Proposition 2 to the pair of the solution of the boundary-value problem (22), (23), and (25) and the Green functions in Table 3 leads to the following:

Proposition 7 (Representation theorem 5) Consider the boundary-value problem (22), (23), and (25c) for an unbounded domain. The outward fluxes of mass, heat, and linear and angular momentums through $\partial D_{\mathrm{g}}$ (i.e., the net fluxes toward the outer far field) are represented in terms of the corresponding Green function:

$$
\begin{array}{r}
{\left[\begin{array}{c}
\mathscr{M}(\infty) \\
\mathscr{Q}(\infty) \\
\mathscr{F}_{\ell}(\infty) \\
\mathscr{T}_{\ell}(\infty)
\end{array}\right]=-\int_{\partial D_{\mathrm{w}}}\left[\begin{array}{c}
P_{\mathrm{w}} u_{n}^{(P ; \infty)}+\tau_{\mathrm{w}} Q_{n}^{(P ; \infty)}-u_{\mathrm{wj}} P_{n j}^{(P ; \infty)} \\
P_{\mathrm{w}} u_{n}^{(T ; \infty)}+\tau_{\mathrm{w}} Q_{n}^{(T ; \infty)}-u_{\mathrm{wj}} P_{n j}^{(T ; \infty)} \\
P_{\mathrm{w}} u_{n}^{(-\ell ; \infty)}+\tau_{\mathrm{w}} Q_{n}^{(-\ell ; \infty)}-u_{\mathrm{wj}} P_{n j}^{(-\ell ; \infty)} \\
P_{\mathrm{w}} u_{n}^{(-\underline{\Omega}(\ell) ; \infty)}+\tau_{\mathrm{w}} Q_{n}^{(-\underline{\Omega}(\ell) ; \infty)}-u_{\mathrm{w} j} P_{n j}^{(-\underline{\Omega}(\ell) ; \infty)}
\end{array}\right] \mathrm{dS}} \\
\\
-\int_{\partial D_{\mathrm{g}}}\left\langle\zeta_{n} h^{-}\left[\begin{array}{l}
G^{(P ; \infty)}-1 \\
G^{(T ; \infty)}-\left(|\zeta|^{2}-\frac{5}{2}\right) \\
G^{(-\ell ; \infty)}+2 \zeta_{j} \ell_{j} \\
G^{(-\underline{\Omega}(\ell) ; \infty)}+2 \zeta_{j} \varepsilon_{j k l} \ell_{k} x_{l}
\end{array}\right]\right\rangle \mathrm{dS},
\end{array}
$$

where $\ell$ is an arbitrary unit vector.

Practically, it is important to note that the above representation yields immediately that of the outward net fluxes through $\partial D_{\mathrm{w}}$ by changing the sign of the right-hand side, because of the conservation of mass, linear and angular momentum, and energy.

\subsection{Reciprocity of the fluxes induced by the Green functions}

As a direct consequence of Propositions 4-7, we obtain a set of corollaries on the reciprocity of the Green functions, which we summarize here. Besides its significance by itself, the reciprocity will play a key role in our entropy theory to be developed in a separate paper. Before showing the corollaries, let us recall the notation convention immediate after (26). For instance, we denote by $\mathscr{M}^{\left(\alpha ; B_{\mathrm{w}}\right)}\left(A_{\mathrm{w}}\right)$ and $\mathscr{Q}^{\left(\alpha ; B_{\mathrm{w}}\right)}\left(A_{\mathrm{w}}\right)$ the dimensionless outward fluxes of mass and heat through area $A_{\mathrm{w}}$ induced by the Green function with respect to area $B_{\mathrm{w}}$ :

$$
\left[\begin{array}{c}
\mathscr{M}^{\left(\alpha ; B_{\mathrm{w}}\right)}\left(A_{\mathrm{w}}\right) \\
\mathscr{Q}^{\left(\alpha ; B_{\mathrm{w}}\right)}\left(A_{\mathrm{w}}\right)
\end{array}\right]=-\int_{A_{\mathrm{w}}}\left[\begin{array}{c}
u_{n}^{\left(\alpha ; B_{\mathrm{w}}\right)}\left(\boldsymbol{x}_{0}\right) \\
Q_{n}^{\left(\alpha ; B_{\mathrm{w}}\right)}\left(\boldsymbol{x}_{0}\right)
\end{array}\right] \mathrm{dS}_{0}, \quad(\alpha=P, T) .
$$

Throughout this subsection, $\boldsymbol{\ell}$ and $\boldsymbol{m}$ are an arbitrary unit vector and the component in their direction will be indicated by subscripted $\ell$ and $m$, e.g., $\mathscr{F}_{\ell}=\mathscr{F}_{j} \ell_{j}, P_{n m}=P_{n j} m_{j}$.

Corollary 2 For any areas $A_{\mathrm{w}}, B_{\mathrm{w}}$ on $\partial D_{\mathrm{w}}$,

$$
\left[\begin{array}{c}
\mathscr{M}^{\left(P ; B_{\mathrm{w}}\right)}\left(A_{\mathrm{w}}\right) \\
\mathscr{Q}^{\left(P ; B_{\mathrm{w}}\right)}\left(A_{\mathrm{w}}\right)
\end{array}\right]=\left[\begin{array}{c}
\mathscr{M}^{\left(P ; A_{\mathrm{w}}\right)}\left(B_{\mathrm{w}}\right) \\
\mathscr{M}^{\left(T ; A_{\mathrm{w}}\right)}\left(B_{\mathrm{w}}\right)
\end{array}\right], \quad\left[\begin{array}{c}
\mathscr{M}^{\left(T ; B_{\mathrm{w}}\right)}\left(A_{\mathrm{w}}\right) \\
\mathscr{Q}^{\left(T ; B_{\mathrm{w}}\right)}\left(A_{\mathrm{w}}\right)
\end{array}\right]=\left[\begin{array}{c}
\mathscr{Q}^{\left(P ; A_{\mathrm{w}}\right)}\left(B_{\mathrm{w}}\right) \\
\mathscr{Q}^{\left(T ; A_{\mathrm{w}}\right)}\left(B_{\mathrm{w}}\right)
\end{array}\right] .
$$


Proof Apply Proposition 4 to the boundary-value problem for $G^{\left(\alpha ; B_{\mathrm{w}}\right)}(\alpha=P, T)$.

Corollary 3 For any $\boldsymbol{x}_{0}, \boldsymbol{x}_{1} \in \partial D_{\mathrm{g}}$ in a bounded domain,

$$
\begin{aligned}
& {\left[\begin{array}{cc}
u_{n}^{\left(P ; \boldsymbol{x}_{0}\right)}\left(\boldsymbol{x}_{1}\right), & u_{n}^{\left(T ; \boldsymbol{x}_{0}\right)}\left(\boldsymbol{x}_{1}\right), u_{n}^{\left(-\boldsymbol{m} ; \boldsymbol{x}_{0}\right)}\left(\boldsymbol{x}_{1}\right) \\
Q_{n}^{\left(P ; \boldsymbol{x}_{0}\right)}\left(\boldsymbol{x}_{1}\right), & Q_{n}^{\left(T ; \boldsymbol{x}_{0}\right)}\left(\boldsymbol{x}_{1}\right), Q_{n}^{\left(-\boldsymbol{m} ; \boldsymbol{x}_{0}\right)}\left(\boldsymbol{x}_{1}\right) \\
P_{n \ell}^{\left(P ; \boldsymbol{x}_{0}\right)}\left(\boldsymbol{x}_{1}\right)-n_{i} \ell_{i} \boldsymbol{\delta}\left(\boldsymbol{x}_{1}-\boldsymbol{x}_{0}\right), & P_{n \ell}^{\left(T ; \boldsymbol{x}_{0}\right)}\left(\boldsymbol{x}_{1}\right), P_{n \ell}^{\left(-\boldsymbol{m} ; \boldsymbol{x}_{0}\right)}\left(\boldsymbol{x}_{1}\right)
\end{array}\right]} \\
& =\left[\begin{array}{ccc}
u_{n}^{\left(P ; \boldsymbol{x}_{1}\right)}\left(\boldsymbol{x}_{0}\right), & Q_{n}^{\left(P ; \boldsymbol{x}_{1}\right)}\left(\boldsymbol{x}_{0}\right), & P_{n m}^{\left(P ; \boldsymbol{x}_{1}\right)}\left(\boldsymbol{x}_{0}\right)-n_{i} m_{i} \boldsymbol{\delta}\left(\boldsymbol{x}_{0}-\boldsymbol{x}_{1}\right) \\
u_{n}^{\left(T ; \boldsymbol{x}_{1}\right)}\left(\boldsymbol{x}_{0}\right), & Q_{n}^{\left(T ; \boldsymbol{x}_{1}\right)}\left(\boldsymbol{x}_{0}\right), & P_{n m}^{\left(T ; \boldsymbol{x}_{1}\right)}\left(\boldsymbol{x}_{0}\right) \\
u_{n}^{\left(-\ell ; \boldsymbol{x}_{1}\right)}\left(\boldsymbol{x}_{0}\right), & Q_{n}^{\left(-\ell ; \boldsymbol{x}_{1}\right)}\left(\boldsymbol{x}_{0}\right), & P_{n m}^{\left(-\ell ; \boldsymbol{x}_{1}\right)}\left(\boldsymbol{x}_{0}\right)
\end{array}\right] .
\end{aligned}
$$

Corollary 4 For any $\boldsymbol{x}_{\mathrm{w}} \in \partial D_{\mathrm{w}}$ and $\boldsymbol{x}_{\mathrm{g}} \in \partial D_{\mathrm{g}}$ for a bounded domain, the following relation holds:

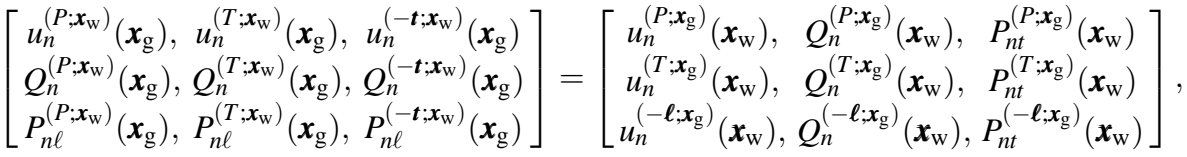

where $\boldsymbol{t}$ is a unit vector tangential to $\partial D_{\mathrm{w}}$ at $\boldsymbol{x}_{\mathrm{w}}$.

Proof Apply Proposition 5 to the boundary-value problem for $G^{\left(\alpha ; x_{0}\right)}(\alpha=P, T,-\boldsymbol{m})$ by putting $\boldsymbol{x}_{\mathrm{g}}=\boldsymbol{x}_{1}$ for Corollary 3 and to that for $G^{\left(\alpha ; \boldsymbol{x}_{\mathrm{w}}\right)}(\alpha=P, T,-\boldsymbol{t})$ for Corollary 4.

Corollary 5 For any areas $A_{\mathrm{g}}, B_{\mathrm{g}} \subseteq \partial D_{\mathrm{g}}$ in a bounded domain,

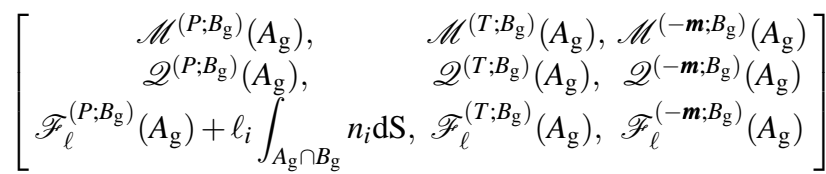

$$
\begin{aligned}
& =\left[\begin{array}{lll}
\mathscr{M}^{\left(P ; A_{\mathrm{g}}\right)}\left(B_{\mathrm{g}}\right), & \mathscr{Q}^{\left(P ; A_{\mathrm{g}}\right)}\left(B_{\mathrm{g}}\right), & \mathscr{F}_{m}^{\left(P ; A_{\mathrm{g}}\right)}\left(B_{\mathrm{g}}\right)+m_{i} \int_{A_{\mathrm{g}} \cap B_{\mathrm{g}}} n_{i} \mathrm{dS} \\
\mathscr{M}^{\left(T ; A_{\mathrm{g}}\right)}\left(B_{\mathrm{g}}\right), & \mathscr{Q}^{\left(T ; A_{\mathrm{g}}\right)}\left(B_{\mathrm{g}}\right), & \mathscr{F}_{m}^{\left(T ; A_{\mathrm{g}}\right)}\left(B_{\mathrm{g}}\right) \\
\mathscr{M}^{\left(-\ell ; A_{\mathrm{g}}\right)}\left(B_{\mathrm{g}}\right), & \mathscr{Q}^{\left(-\ell ; A_{\mathrm{g}}\right)}\left(B_{\mathrm{g}}\right), & \mathscr{F}_{m}^{\left(-\ell ; A_{\mathrm{g}}\right)}\left(B_{\mathrm{g}}\right)
\end{array}\right] .
\end{aligned}
$$

Corollary 6 For any areas $A_{\mathrm{w}} \in \partial D_{\mathrm{w}}$ and $A_{\mathrm{g}} \in \partial D_{\mathrm{g}}$ in a bounded domain,

$$
\left[\begin{array}{cc}
\mathscr{M}^{\left(P ; A_{\mathrm{w}}\right)}\left(A_{\mathrm{g}}\right), \mathscr{M}^{\left(T ; A_{\mathrm{w}}\right)}\left(A_{\mathrm{g}}\right) \\
\mathscr{Q}^{\left(P ; A_{\mathrm{w}}\right)}\left(A_{\mathrm{g}}\right), & \mathscr{Q}^{\left(T ; A_{\mathrm{w}}\right)}\left(A_{\mathrm{g}}\right) \\
\mathscr{F}_{\ell}^{\left(P ; A_{\mathrm{w}}\right)}\left(A_{\mathrm{g}}\right), & \mathscr{F}_{\ell}^{\left(T ; A_{\mathrm{w}}\right)}\left(A_{\mathrm{g}}\right)
\end{array}\right]=\left[\begin{array}{cc}
\mathscr{M}^{\left(P ; A_{\mathrm{g}}\right)}\left(A_{\mathrm{w}}\right), & \mathscr{Q}^{\left(P ; A_{\mathrm{g}}\right)}\left(A_{\mathrm{w}}\right) \\
\mathscr{M}^{\left(T ; A_{\mathrm{g}}\right)}\left(A_{\mathrm{w}}\right), & \mathscr{Q}^{\left(T ; A_{\mathrm{g}}\right)}\left(A_{\mathrm{w}}\right) \\
\mathscr{M}^{\left(-\ell ; A_{\mathrm{g}}\right)}\left(A_{\mathrm{w}}\right), & \mathscr{Q}^{\left(-\ell ; A_{\mathrm{g}}\right)}\left(A_{\mathrm{w}}\right)
\end{array}\right] .
$$

Proof Apply Proposition 6 to the boundary-value problem for $G^{\left(\alpha ; B_{\mathrm{g}}\right)}(\alpha=P, T,-\boldsymbol{m})$ for Corollary 5 and to that for $G^{\left(\alpha ; A_{\mathrm{w}}\right)}(\alpha=P, T)$ for Corollary 6 .

Corollary 7 The following reciprocal relation holds:

$$
\begin{aligned}
\mathscr{M}^{(T ; \infty)}(\infty) & =\mathscr{Q}^{(P ; \infty)}(\infty), & \mathscr{M}^{(-\boldsymbol{m} ; \infty)}(\infty)=\mathscr{F}_{m}^{(P ; \infty)}(\infty), \\
\mathscr{Q}^{(-\boldsymbol{m} ; \infty)}(\infty) & =\mathscr{F}_{m}^{(T ; \infty)}(\infty), & \mathscr{F}_{\ell}^{(-\boldsymbol{m} ; \infty)}(\infty)=\mathscr{F}_{m}^{(-\ell ; \infty)}(\infty), \\
\mathscr{M}^{(-\underline{\Omega}(\boldsymbol{m}) ; \infty)}(\infty) & =\mathscr{T}_{m}^{(P ; \infty)}(\infty), & \mathscr{F}_{\ell}^{(-\underline{\Omega}(\boldsymbol{m}) ; \infty)}(\infty)=\mathscr{T}_{m}^{(-\ell ; \infty)}(\infty), \\
\mathscr{Q}^{(-\underline{\Omega}(\boldsymbol{m}) ; \infty)}(\infty) & =\mathscr{T}_{m}^{(T ; \infty)}(\infty), & \mathscr{T}_{\ell}^{(-\underline{\Omega}(\boldsymbol{m}) ; \infty)}(\infty)=\mathscr{T}_{m}^{(-\underline{\Omega}(\ell) ; \infty)}(\infty) .
\end{aligned}
$$


Corollary 8 For any $\boldsymbol{x} \in \partial D_{\mathrm{w}}$ and $A \subseteq \partial D_{\mathrm{w}}$,

$$
\begin{aligned}
& {\left[\begin{array}{ccc}
\mathscr{M}^{(P ; \boldsymbol{x})}(\infty), & \mathscr{M}^{(T ; \boldsymbol{x})}(\infty), & \mathscr{M}^{(-\boldsymbol{m} ; \boldsymbol{x})}(\infty) \\
\mathscr{Q}^{(P ; \boldsymbol{x})}(\infty), & \mathscr{Q}^{(T ; \boldsymbol{x})}(\infty), & \mathscr{Q}^{(-\boldsymbol{m} ; \boldsymbol{x})}(\infty) \\
\mathscr{F}_{\ell}^{(P ; \boldsymbol{x})}(\infty), & \mathscr{F}_{\ell}^{(T ; \boldsymbol{x})}(\infty), & \mathscr{F}_{\ell}^{(-\boldsymbol{m} ; \boldsymbol{x})}(\infty) \\
\mathscr{T}_{\ell}^{(P ; \boldsymbol{x})}(\infty), & \mathscr{T}_{\ell}^{(T ; \boldsymbol{x})}(\infty), & \mathscr{T}_{\ell}^{(-\boldsymbol{m} ; \boldsymbol{x})}(\infty)
\end{array}\right]=-\left[\begin{array}{ccc}
u_{n}^{(P ; \infty)}(\boldsymbol{x}), & Q_{n}^{(P ; \infty)}(\boldsymbol{x}), & P_{n m}^{(P ; \infty)}(\boldsymbol{x}) \\
u_{n}^{(T ; \infty)}(\boldsymbol{x}), & Q_{n}^{(T ; \infty)}(\boldsymbol{x}), & P_{n m}^{(T ; \infty)}(\boldsymbol{x}) \\
u_{n}^{(-\ell ; \infty)}(\boldsymbol{x}), & Q_{n}^{(-\ell ; \infty)}(\boldsymbol{x}), & P_{n m}^{(-\ell ; \infty)}(\boldsymbol{x}) \\
u_{n}^{(-\underline{\Omega}(\boldsymbol{\ell}) ; \infty)}(\boldsymbol{x}), & Q_{n}^{(-\underline{\Omega}(\boldsymbol{\ell}) ; \infty)}(\boldsymbol{x}), P_{n m}^{(-\underline{\Omega}(\ell) ; \infty)}(\boldsymbol{x})
\end{array}\right],} \\
& {\left[\begin{array}{cc}
\mathscr{M}^{(P ; A)}(\infty), & \mathscr{M}^{(T ; A)}(\infty) \\
\mathscr{Q}^{(P ; A)}(\infty), & \mathscr{Q}^{(T ; A)}(\infty) \\
\mathscr{F}_{\ell}^{(P ; A)}(\infty), & \mathscr{F}^{(T ; A)}(\infty) \\
\mathscr{T}_{\ell}^{(P ; A)}(\infty), & \mathscr{T}_{\ell}^{(T ; A)}(\infty)
\end{array}\right]=\left[\begin{array}{cc}
\mathscr{M}^{(P ; \infty)}(A), & \mathscr{Q}^{(P ; \infty)}(A) \\
\mathscr{M}^{(T ; \infty)}(A), & \mathscr{Q}^{(T ; \infty)}(A) \\
\mathscr{M}^{(-\ell ; \infty)}(A), & \mathscr{Q}^{(-\ell ; \infty)}(A) \\
\mathscr{M}^{(-\underline{\Omega}(\ell) ; \infty)}(A), & \mathscr{Q}^{(-\underline{\Omega}(\ell) ; \infty)}(A)
\end{array}\right]}
\end{aligned}
$$

Proof Apply Proposition 7 to the boundary-value problem for $G^{(\alpha ; \infty)}(\alpha=T,-\boldsymbol{m},-\underline{\Omega}(\boldsymbol{m}))$ for Corollary 7 and to that for $G^{(\alpha ; x)}(\alpha=P, T,-\boldsymbol{m})$ or $G^{(\beta ; A)}(\beta=P, T)$ for Corollary 8.

\section{Applications of the representation theorem}

In this section, we present application examples of the representation theorem. Some of them (Examples 1, 2, and 5) have been discussed in the literature as the examples of the Onsager-Casimir reciprocity in the connection to the entropy production (e.g., [2,42, 8, 43, 9]). It should be noted that they are recovered merely as byproducts of the representation theorem and that the discussion of the entropy production is entirely excluded from the present paper.

5.1 Mass and heat fluxes and the force acting on the resting bodies in an unbounded domain

Consider a group of $N$ resting bodies (say, $B_{1}, \ldots, B_{N}$ ) arranged in a finite region in the gas occupying an unbounded domain. In a far field, the gas is in the equilibrium state at rest with density $\rho_{0}$ and temperature $T_{0}$. The respective bodies in the group may be a simple solid body or a condensed phase of the gas; they do not change in time their shape and surface temperature and have no surface velocity.

When the deviation from the reference equilibrium state is small, the problem can be linearized around the reference state. Then, as a direct application of Proposition 4 and Corollary $2\left(\partial D_{\mathrm{w}}=\partial B_{1}+\cdots \partial B_{N}, \partial D_{\mathrm{g}}=\partial D_{\mathrm{g}}^{(\mathrm{ii})}, A_{\mathrm{w}}=\partial B_{j}, B_{\mathrm{w}}=\partial B_{k}, u_{\mathrm{w} i}=0\right.$, and $\left.h=0\right)$, we have the following statement for the mass and heat transfered to the resting bodies:

Corollary 9 (mass and heat transfered to the resting bodies) The mass and heat transfered to the body $B_{j}(j=1, \ldots, N)$ per unit time can be expressed by the mass and heat flow distributions over the whole body surface $\partial D_{\mathrm{w}}$ induced by the Green functions for the pressure and temperature sources with respect to $\partial B_{j}$ :

$$
\left[\begin{array}{c}
\mathscr{M}\left(\partial B_{j}\right) \\
\mathscr{Q}\left(\partial B_{j}\right)
\end{array}\right]=-\int_{\partial D_{\mathrm{w}}}\left[\begin{array}{c}
u_{n}^{\left(P ; \partial B_{j}\right)}(\boldsymbol{x}), Q_{n}^{\left(P ; \partial B_{j}\right)}(\boldsymbol{x}) \\
u_{n}^{\left(T ; \partial B_{j}\right)}(\boldsymbol{x}), Q_{n}^{\left(T ; \partial B_{j}\right)}(\boldsymbol{x})
\end{array}\right]\left[\begin{array}{c}
P_{\mathrm{w}}(\boldsymbol{x}) \\
\tau_{\mathrm{w}}(\boldsymbol{x})
\end{array}\right] \mathrm{dS} .
$$

In particular, 
(i) if there is no condensed phase in the group of bodies, the mass transfered to each body vanishes and the above expression becomes much simpler as ${ }^{16}$

$$
\mathscr{Q}\left(\partial B_{j}\right)=-\int_{\partial D_{\mathrm{w}}} Q_{n}^{\left(T ; \partial B_{j}\right)}(\boldsymbol{x}) \tau_{\mathrm{w}}(\boldsymbol{x}) \mathrm{dS} .
$$

(ii) the following reciprocity holds:

$$
\left[\begin{array}{cc}
\mathscr{M}^{\left(P ; \partial B_{k}\right)}\left(\partial B_{j}\right), \mathscr{M}^{\left(T ; \partial B_{k}\right)}\left(\partial B_{j}\right) \\
\mathscr{Q}^{\left(P ; \partial B_{k}\right)}\left(\partial B_{j}\right), & \mathscr{Q}^{\left(T ; \partial B_{k}\right)}\left(\partial B_{j}\right)
\end{array}\right]=\left[\begin{array}{l}
\mathscr{M}^{\left(P ; \partial B_{j}\right)}\left(\partial B_{k}\right), \mathscr{Q}^{\left(P ; \partial B_{j}\right)}\left(\partial B_{k}\right) \\
\mathscr{M}^{\left(T ; \partial B_{j}\right)}\left(\partial B_{k}\right), \mathscr{Q}^{\left(T ; \partial B_{j}\right)}\left(\partial B_{k}\right)
\end{array}\right] .
$$

Example 1 (Evaporation from and condensation onto a volatile sphere) Consider the case where the group of the bodies is a single spherical condensed phase of the gas (for short, a volatile sphere). Denoting by $S$ the surface of the volatile sphere, we have the expression

$$
\left[\begin{array}{c}
\mathscr{M}(S) \\
\mathscr{Q}(S)
\end{array}\right]=-\int_{S}\left[\begin{array}{l}
u_{n}^{(P ; S)}(\boldsymbol{x}), Q_{n}^{(P ; S)}(\boldsymbol{x}) \\
u_{n}^{(T ; S)}(\boldsymbol{x}), Q_{n}^{(T ; S)}(\boldsymbol{x})
\end{array}\right]\left[\begin{array}{c}
P_{\mathrm{w}}(\boldsymbol{x}) \\
\tau_{\mathrm{w}}(\boldsymbol{x})
\end{array}\right] \mathrm{dS}
$$

and the reciprocity

$$
\mathscr{M}^{(T ; S)}(S)=\mathscr{Q}^{(P ; S)}(S) .
$$

Noted that $\mathscr{M}^{(P ; S)}$ and $\mathscr{Q}^{(P ; S)}$ are the dimensionless mass and heat passing through a point on $S$ per unit time and area for $P_{\mathrm{w}}=1$ and $\tau_{\mathrm{w}}=0$, while $\mathscr{M}^{(T ; S)}$ and $\mathscr{Q}^{(T ; S)}$ are those for $P_{\mathrm{w}}=0$ and $\tau_{\mathrm{w}}=1$. Thus, as far as the mass and heat transfer is concerned, the general result is readily obtained by the study of two elemental situations, $P_{\mathrm{w}}=1, \tau_{\mathrm{w}}=0$ and $P_{\mathrm{w}}=0, \tau_{\mathrm{w}}=$ 1. The above reciprocity was numerically verified in the literature (e.g., $[42,43])$.

We also have a statement on the force and torque on the group of resting bodies (not individual bodies) as a consequence of Proposition 7 and the conservation of linear and angular momentums:

Corollary 10 (Force and torque on the group of resting bodies) The force and torque on the group of bodies can be obtained from the mass and heat flow distributions over the whole body surface $\partial D_{\mathrm{w}}$ induced by the Green functions for velocity and rotation sources in a far field. If we denote by $p_{0} L^{2} \mathscr{F}_{\ell}\left(\partial D_{\mathrm{w}}\right)$ and $p_{0} L^{3} \mathscr{T}_{\ell}\left(\partial D_{\mathrm{w}}\right)$ the force and torque on the group of bodies in the $\ell$-direction. they are expressed as

$$
\left[\begin{array}{c}
\mathscr{F}_{\ell}\left(\partial D_{\mathrm{w}}\right) \\
\mathscr{T}_{\ell}\left(\partial D_{\mathrm{w}}\right)
\end{array}\right]=-\int_{\partial D_{\mathrm{w}}}\left(P_{\mathrm{w}}(\boldsymbol{x})\left[\begin{array}{c}
u_{n}^{(\ell ; \infty)}(\boldsymbol{x}) \\
u_{n}^{(\underline{\Omega}(\ell) ; \infty)}(\boldsymbol{x})
\end{array}\right]+\tau_{\mathrm{w}}(\boldsymbol{x})\left[\begin{array}{c}
Q_{n}^{(\ell ; \infty)}(\boldsymbol{x}) \\
Q_{n}^{(\underline{\Omega(\ell) ; \infty)}}(\boldsymbol{x})
\end{array}\right]\right) \mathrm{d} S .
$$

${ }^{16}$ Consider a temperature field in the fluid dynamic limit, which is described by the Laplace equation. Let us denote by $\tau$ the perturbed temperature of the gas and by $\tau^{\left(\partial B_{j}\right)}$ the counterpart when the temperature source is put on $\partial B_{j}$, i.e., $\Delta \tau=0, \Delta \tau^{\left(\partial B_{j}\right)}=0, \tau=\tau_{\mathrm{w}}$ on $\partial D_{\mathrm{w}}, \tau^{\left(\partial B_{j}\right)}=1$ on $\partial B_{j}$, and $\tau^{\left(\partial B_{j}\right)}=0$ on $\partial D_{\mathrm{w}} \backslash \partial B_{j}$. Then, the Green formula $\int_{D}\left(\tau \Delta \tau^{\left(\partial B_{j}\right)}-\tau^{\left(\partial B_{j}\right)} \Delta \tau\right) \mathrm{d} \boldsymbol{x}=\int_{\partial D}\left(\tau \nabla \tau^{\left(\partial B_{j}\right)}-\tau^{\left(\partial B_{j}\right)} \nabla \tau\right) \cdot \boldsymbol{n} \mathrm{d} S$ is reduced to

$$
0=\int_{\partial D_{\mathrm{w}}} \tau_{\mathrm{w}} \nabla \tau^{\left(\partial B_{j}\right)} \cdot \boldsymbol{n} \mathrm{d} \mathrm{S}-\int_{\partial B_{j}} \nabla \tau \cdot \boldsymbol{n} \mathrm{d} \mathrm{S}+\int_{\partial D_{\mathrm{g}}}\left(\tau \boldsymbol{\nabla} \tau^{\left(\partial B_{j}\right)}-\tau^{\left(\partial B_{j}\right)} \nabla \tau\right) \cdot \boldsymbol{n} \mathrm{d} \mathrm{S} .
$$

Since $\tau$ and $\tau^{\left(\partial B_{j}\right)}$ decays with the rate of $|\boldsymbol{x}|^{-1}$ as $|\boldsymbol{x}| \rightarrow \infty$, the third integral vanishes, and we have

$$
\int_{\partial D_{\mathrm{w}}} \tau_{\mathrm{w}} \nabla \tau^{\left(\partial B_{j}\right)} \cdot \boldsymbol{n} \mathrm{dS}=\int_{\partial B_{j}} \nabla \tau \cdot \boldsymbol{n} \mathrm{dS} .
$$

The expression (29) is the generalization of this relation to the gas of arbitrary Knudsen number. 
In particular, when there is no condensed phase in the group, i.e., when $\partial D_{\mathrm{w}}$ is a simple boundary, the mass transfered to each body vanishes and the force and torque on the group of bodies can be expressed only by the heat flow distribution:

$$
\left[\begin{array}{c}
\mathscr{F}_{\ell}\left(\partial D_{\mathrm{w}}\right) \\
\mathscr{T}_{\ell}\left(\partial D_{\mathrm{w}}\right)
\end{array}\right]=-\int_{\partial D_{\mathrm{w}}} \tau_{\mathrm{w}}(\boldsymbol{x})\left[\begin{array}{c}
Q_{n}^{(\ell ; \infty)}(\boldsymbol{x}) \\
Q_{n}^{(\underline{\Omega}(\boldsymbol{\ell}) ; \infty)}(\boldsymbol{x})
\end{array}\right] \mathrm{dS} .
$$

Proof By the linear and angular momentum conservations, $\mathscr{F}_{\ell}\left(\partial D_{\mathrm{w}}\right)=-\mathscr{F}_{\ell}(\infty)$ and $\mathscr{T}_{\ell}\left(\partial D_{\mathrm{w}}\right)=$ $-\mathscr{T}_{\ell}(\infty)$. Use Proposition 7 for the representation of $\mathscr{F}_{\ell}(\infty)$ and $\mathscr{T}_{\ell}(\infty)$ by putting $u_{\mathrm{w} i}=0$ and $h=0$. Finally use the parity of $G^{(\ell ; \infty)}$ in Table 3 .

Example 2 (Force acting on a simple solid body with non-uniform temperature) [44] Consider the case where the group of the bodies is a single simple solid body. Denoting by $\mathrm{S}$ the surface of the body, the dimensionless force acting on the body is given by

$$
\mathscr{F}_{\ell}(\mathrm{S})=-\int_{\mathrm{S}} \tau_{\mathrm{w}}(\boldsymbol{x}) Q_{n}^{(\ell ; \infty)}(\boldsymbol{x}) \mathrm{dS} .
$$

$Q_{n}^{(\ell ; \infty)}$ is the dimensionless heat flow induced by $G^{(\ell ; \infty)}$. This Green function is a solution of the problem of a uniform slow flow in the $\ell$-direction past the body with the reference uniform upstream temperature $T_{0}$ (to be precise, the solution normalized by the upstream flow speed).

Extension of Corollaries 9 and 10 to the case of bodies with moving surface under the constraint of $\boldsymbol{u}_{w} \cdot \boldsymbol{n}=0$ is straightforward. In the case of a bounded domain with the boundary composed only of the real boundary $\left(\partial D=\partial D_{\mathrm{w}}\right)$, Corollaries 9 and 10 hold as it is with a proper choice of the reference equilibrium state. On the other hand, if the gas is not necessarily in some resting equilibrium state in a far field, we need to use Propositions 4 or 7 directly. The next is such an example.

Example 3 (Thermophoresis) Consider an infinite expanse of a resting gas with the temperature distribution $T_{0}\left(1+C x_{1}\right)$ (C: a positive constant), in which a single simple solid body with temperature $T_{0}$ is located at the origin. In this situation, there occurs a force acting on the body, which is the present concern (see, e.g., [5-7] and the references therein). The sources on the boundary are given by $g_{\mathrm{w}}=0$ and $h=C\left[\left(|\boldsymbol{\zeta}|^{2}-\frac{5}{2}\right) x_{1}-(\sqrt{\pi} / 2) \operatorname{Kn} \zeta_{1} A(|\boldsymbol{\zeta}|)\right]$ in this situation, where $A$ is the one already defined in Sect. 3.1. We denote by $\mathrm{S}$ the surface of the body and use Proposition 7 to obtain the relation

$$
\mathscr{F}_{\ell}(\mathrm{S})=-\mathscr{F}_{\ell}(\infty)=-C \int_{\partial D_{\mathrm{g}}}\left(x_{1} Q_{n}^{(\ell ; \infty)}+\frac{\sqrt{\pi}}{2} \mathrm{Kn}\left\langle\zeta_{n} \zeta_{1} A(|\zeta|) G^{(\ell ; \infty)}\right\rangle\right) \mathrm{d} \mathrm{S} .
$$

Here the parity of $G^{(\ell ; \infty)}$ in Table 3 and the conservation of momentum have been used.

In the meantime, since both $h$ and $G^{(\ell ; \infty)}$ solve (22), we can show in the same way as in the proof of Proposition 1 that $\int_{\partial D}\left\langle\zeta_{n} h^{-} G^{(\ell ; \infty)}\right\rangle \mathrm{dS}=0$. By using this fact and $\partial D=\partial D_{\mathrm{g}} \cup \mathrm{S}$, we obtain an alternative expression ${ }^{17}$

$$
\mathscr{F}_{\ell}(S)=C \int_{\partial D_{\mathrm{w}}}\left(x_{1} Q_{n}^{(\ell ; \infty)}+\frac{\sqrt{\pi}}{2} \operatorname{Kn}\left\langle\zeta_{n} \zeta_{1} A(|\zeta|) G^{(\ell ; \infty)}\right\rangle\right) \mathrm{d} S
$$

17 Our result is different from that in [8]. This is due to the fact that the term of $\zeta_{1} A(|\zeta|)$ is missing in the asymptotic form of the perturbed distribution function in that reference. The expression in [8] is incorrect. 


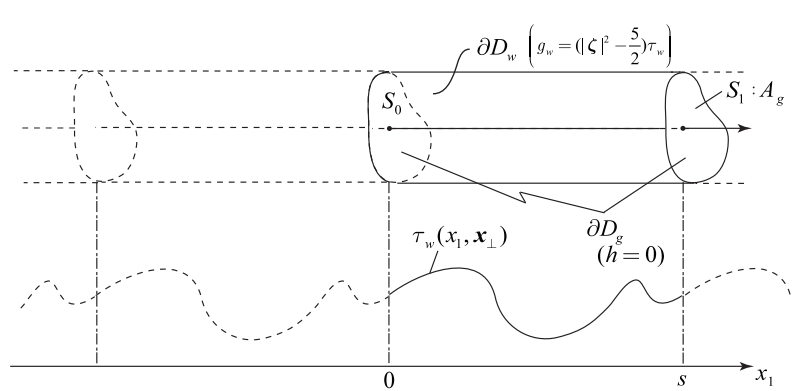

Fig. 2 A straight pipe with a periodic temperature distribution.

\subsection{Mass and heat fluxes along various channels}

Example 4 (Golse's theorem) Consider a gas in a straight pipe with a uniform cross-section. The temperature of the pipe is periodic and is constant in time. In the situation, a steady flow is induced in the gas by the non-uniformity of the temperature of the pipe. Golse proved that the induced flow field does not generate the mass flux through the pipe cross-section, by assuming that the temperature variation is so small that the linearization of the problem is allowed (Golse's theorem [12]). We shall show below that we can reach the same conclusion with a slight extension of the statement by the application of Proposition 6 .

Let us take the coordinate $x_{1}$ in the axial direction of the pipe as in Fig. 2. Let $s$ be the period in this direction and $D$ be the domain surrounded by the pipe wall and by the crosssections $S_{0}$ and $S_{1}$ located respectively at $x_{1}=0$ and $x_{1}=s$. Because $P_{\mathrm{w}}=0, \boldsymbol{u}_{\mathrm{w}}=0$, and $h=0$, Proposition 6 yields, by putting $A_{\mathrm{g}}=S_{1}$, the relation

$$
\mathscr{M}\left(S_{1}\right)=-\int_{\partial D_{\mathrm{w}}} \tau_{\mathrm{w}}\left(x_{1}, \boldsymbol{x}_{\perp}\right) Q_{n}^{\left(P ; S_{1}\right)}\left(x_{1}, \boldsymbol{x}_{\perp}\right) \mathrm{d} \mathrm{S},
$$

where $\boldsymbol{x}_{\perp}=\left(x_{2}, x_{3}\right)$, and here the Green function $G^{\left(P ; S_{1}\right)}$ is the solution of the problem:

$$
\begin{aligned}
\zeta_{i} \frac{\partial G^{\left(P ; S_{1}\right)}}{\partial x_{i}}=\frac{2}{\sqrt{\pi}} \frac{1}{\mathrm{Kn}} \mathscr{L}\left(G^{\left(P ; S_{1}\right)}\right), & \\
G^{\left(P ; S_{1}\right)}\left(x_{1}, \boldsymbol{x}_{\perp}, \boldsymbol{\zeta}\right)=\int_{\zeta_{n}^{*}<0} \frac{\left|\boldsymbol{\zeta}_{n}^{*}\right| E\left(\boldsymbol{\zeta}^{*}\right)}{\left|\zeta_{n}\right| E(\boldsymbol{\zeta})} R_{\mathrm{CR}}\left(\boldsymbol{\zeta}^{*}, \boldsymbol{\zeta}\right) G^{\left(P ; S_{1}\right)}\left(x_{1}, \boldsymbol{x}_{\perp}, \boldsymbol{\zeta}^{*}\right) \mathrm{d} \boldsymbol{\zeta}^{*} & \zeta_{n}>0,\left(x_{1}, \boldsymbol{x}_{\perp}\right) \in \partial D_{\mathrm{w}} \\
G^{\left(P ; S_{1}\right)}\left(s, \boldsymbol{x}_{\perp}, \boldsymbol{\zeta}\right)=1+G^{\left(P ; S_{1}\right)}\left(0, \boldsymbol{x}_{\perp}, \boldsymbol{\zeta}\right) & \text { for } \zeta_{1}<0, \\
G^{\left(P ; S_{1}\right)}\left(0, \boldsymbol{x}_{\perp}, \boldsymbol{\zeta}\right)=G^{\left(P ; S_{1}\right)}\left(s, \boldsymbol{x}_{\perp}, \boldsymbol{\zeta}\right)-1 & \text { for } \zeta_{1}>0
\end{aligned}
$$

where $R_{\mathrm{CR}}$ is independent of $\boldsymbol{x}$. If the pipe wall is of the locally isotropic boundary [12,10], we can seek the solution in the form ${ }^{18} G^{\left(P ; S_{1}\right)}=x_{1} / s+\zeta_{1} \Phi\left(x_{\perp}, \boldsymbol{\zeta}\right)$, where $\Phi$ is even with respect to $\zeta_{1}$. Since $\Phi$ is even, $Q_{n}^{\left(P ; S_{1}\right)}\left(=\left\langle\zeta_{n} G^{\left(P ; S_{1}\right)}\right\rangle\right)$ vanishes, so that $\mathscr{M}\left(S_{1}\right)=0$.

In the proof by Golse in the Appendix A.4 of [12], the Maxwell-type boundary condition with $\tau_{\mathrm{w}}$ depending only on $x_{1}$ is considered. In contrast, we arrived at the same conclusion for a more general boundary condition and $\tau_{\mathrm{w}}$. In the sense, the present approach provides a slight extension of the applicable range of his statement.

\footnotetext{
18 Here we assumed the similarity solution for brevity. Actually, however, this assumption is not necessary and we can show $Q_{n}^{\left(P ; S_{1}\right)}=0$ by the argument similar to that by Golse for the reduced problem (30).
} 


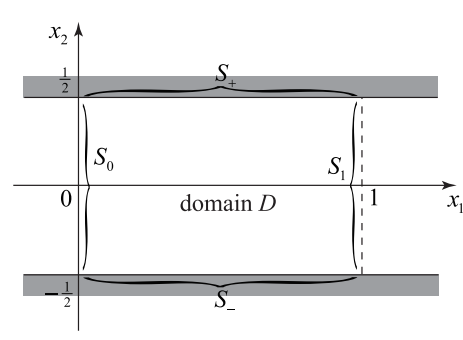

Fig. 3 The channel between two parallel infinite plates and the domain $D$ for the application of the representation theorem.

Example 5 (Poiseuille, thermal transpiration, and Couette flows) Consider the steady behavior of the gas between two parallel plates located at $x_{2}= \pm \frac{1}{2}$ in the following cases (see, e.g., [45-47]):

1. Poiseuille flow (PF, for short): two plates are at rest with a uniform constant temperature $T_{0}$, and a uniform gradient of pressure in the $x_{1}$-direction is imposed, i.e., the gas pressure is given by $p_{0}\left(1+C_{\mathrm{P}} x_{1}\right)$ with $C_{\mathrm{P}}$ being constant.

2. Thermal transpiration (TT, for short): two plates are at rest with a common temperature distribution $T_{0}\left(1+C_{\mathrm{T}} x_{1}\right)$ with $C_{\mathrm{T}}$ being constant.

3. Couette flow (CF, for short): two plates are at a uniform constant temperature $T_{0}$. The upper plate $\left(x_{2}=\frac{1}{2}\right)$ is moving with the velocity $\left(\left(2 k T_{0} / m\right)^{1 / 2} u_{\mathrm{w} 1}, 0,0\right)$, while the lower $\left(x_{2}=-\frac{1}{2}\right)$ is at rest.

We assume that $\left|C_{\mathrm{P}}\right|,\left|C_{\mathrm{T}}\right|$, and $\left|u_{\mathrm{w} 1}\right|$ are so small that the problems can be linearized around the resting equilibrium state with temperature $T_{0}$ and pressure $p_{0}$. Further the state is assumed to be independent in $x_{3}$. In what follows, we denote by putting the subscript PF, TT, and CF the solutions of the problems 1-3 normalized by the constants $C_{\mathrm{P}}, C_{\mathrm{T}}$, and $u_{\mathrm{w} 1}$.

Let $D$ be the domain defined by $0<x_{1}<1,-\frac{1}{2}<x_{2}<\frac{1}{2}$, and $0<x_{3}<1$. (see Fig. 3) Let $S_{0}$ and $S_{1}$ the cross-section (per unit length in the $x_{3}$-direction) of the channel at $x_{1}=0$ and $x_{1}=1$. Let $S_{ \pm}$be the surface of the plates at $x_{2}= \pm \frac{1}{2}$ in the range of $0<x_{1}<1$ and $0<x_{3}<1$. It is easy to check that the solution of problem TT solves the problem in $D$ with $g_{\mathrm{w}}=x_{1}\left(|\zeta|^{2}-\frac{5}{2}\right), h\left(x_{1}=0\right)=0$, and $h\left(x_{1}=1\right)=|\zeta|^{2}-\frac{5}{2}$, while that of problem CF solves the problem in $D$ with $g_{\mathrm{w}}=2 \zeta_{1} \delta\left(x_{2}-\frac{1}{2}\right)$ and $h=0$. With these in mind, we apply Propositon 6 by putting $A_{\mathrm{g}}=S_{1}$ to obtain

$$
\mathscr{M}_{\mathrm{TT}}\left(S_{1}\right)= \pm \int_{S_{ \pm}} x_{1} Q_{2}^{\left(P ; S_{1}\right)} \mathrm{dS}+\mathscr{Q}^{\left(P ; S_{1}\right)}\left(S_{1}\right), \quad \mathscr{M}_{\mathrm{CF}}\left(S_{1}\right)=-\mathscr{F}_{1}^{\left(P ; S_{1}\right)}\left(S_{+}\right) .
$$

As in Example 4, we assume the plates are of the locally isotropic boundary, so that the Green function $G^{\left(P ; S_{1}\right)}$ is given in the form of $G^{\left(P ; S_{1}\right)}=x_{1}+\zeta_{1} \Phi\left(x_{2}, \zeta_{2},|\zeta|\right)$. Since $\Phi$ is even in $\zeta_{1}, Q_{2}^{\left(P ; S_{1}\right)}$ in the first equality vanishes. Finally noting that the normalized solution of the Poiseuille flow problem is the Green function $G^{\left(P ; S_{1}\right)}$, we can rewrite the above relations as

$$
\mathscr{M}_{\mathrm{TT}}\left(S_{1}\right)=\mathscr{Q}_{\mathrm{PF}}\left(S_{1}\right), \quad \mathscr{M}_{\mathrm{CF}}\left(S_{1}\right)=-\mathscr{F}_{1 \mathrm{PF}}\left(S_{+}\right),
$$

which show the cross relations among the three basic flows (see, e.g., [2]).

Example 6 (Thermal pumps) Consider a gas in the pipe with periodic ditches as in Fig. 4(a). The temperature distribution of the pipe surface is constant in time and is periodic in $x_{1}$ with the same period $s$ as the geometric structure. It is known that a one-way flow is induced by the non-uniform temperature in the pipe [48], which is the driving mechanism of the socalled Knudsen pump $[49,10]$. When the perturbed temperature of the pipe surface is small 


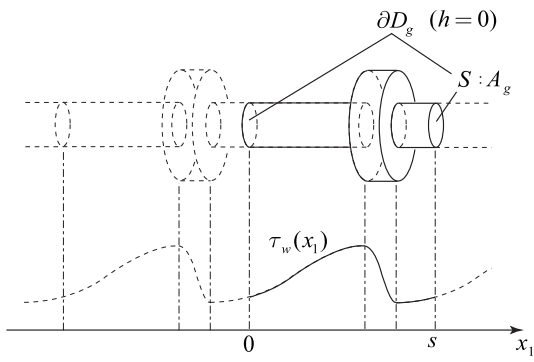

(a) Knudsen pump

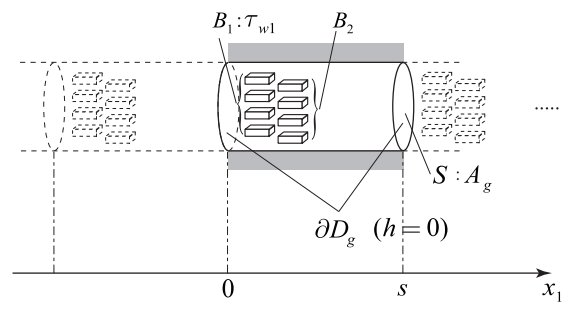

(b) Thermal edge pump

Fig. 4 Schematics of thermal pumps.

enough, we can apply Proposition 6 to the gas domain $D$ (the unit stage of the pump drawn in solid lines in the figure) to have the relation

$$
\mathscr{M}(S)=-\int_{\partial D_{\mathrm{w}}} \tau_{\mathrm{w}}(\boldsymbol{x}) Q_{n}^{(P ; S)}(\boldsymbol{x}) \mathrm{dS},
$$

because $P_{\mathrm{w}}=0, \boldsymbol{u}_{\mathrm{w}}=0$, and $h=0$, where $S$ is the cross-section at $x_{1}=s$. Thus the mass flux by the one-way flow induced in the pump can be expressed by the heat flow distribution on the pipe surface of the Green function $G^{(P ; S)}$ for the pressure source on $S$.

Even when the pipe is a simple straight one with a uniform temperature distribution, a one-way flow can be induced if two arrays of uniformly heated and unheated plates, say $B_{1}$ and $B_{2}$, are put inside the channel periodically with respect to $x_{1}$ with period $s$ [see Fig. 4(b)]. The pipe equipped with such arrays of plates is called the thermal edge pump $[50,10]$. For simplicity, let the temperature of the array $B_{2}$ be the same as the pipe temperature $T_{0}$. Then, if the uniform perturbed temperature $\tau_{\mathrm{w} 1}$ of the array $B_{1}$ is small enough, we can apply Proposition 6 to the gas domain $D$ (the unit stage of the pump drawn in solid lines in the figure) to have the relation

$$
\mathscr{M}(S)=\tau_{\mathrm{w}_{1}} \mathscr{Q}^{(P ; S)}\left(\partial B_{1}\right),
$$

because $\tau_{\mathrm{w}}(\boldsymbol{x})=\tau_{\mathrm{w} 1}$ on $\partial B_{1}$ and $\tau_{\mathrm{w}}=0$ both on $\partial B_{2}$ and on the pipe surface, $P_{\mathrm{w}}=0$, $\boldsymbol{u}_{\mathrm{w}}=0$, and $h=0$, where $S$ is the cross-section at $x_{1}=s$. Thus the mass flux by the one-way flow induced in the pump can be obtained by knowing the heat that the array $B_{1}$ receives in the flow of the Green function $G^{(P ; S)}$ for the pressure source on $S$.

\section{Conclusion}

In the present paper, we first established a symmetric relation (14) that holds widely between two steady problems of the linearized Boltzmann equation in Sect. 2. We also presented a concrete set of situations where the required condition is seen to be fulfilled by a rather simple argument: the bounded domain, one-dimensional half-space, and three dimensional unbounded domain with a finite confinement of bodies. Then, in Sect. 3, we showed some application examples to the second situation (one-dimensional half-space problems). In particular, four unknown relations among the Knudsen layer problems were newly obtained. 
A remarkable difference of the first and third situations from the second was the fact that we may deal with the source on the boundary separately in the former. Making use of this property, we developed in Sect. 4 a unified approach to the first and third situations, on the basis of the symmetric relation, to establish general expressions of the mass, linear and angular momentum, and heat fluxes, which we called the representation theorem. The theorem tells that the problem of finding a flux on the boundary is reduced to finding the solution of a single elemental problem in the considered domain, which we called the Green function, the response of the gas system to a proper elemental disturbance from the surroundings. These disturbances are the pressure, velocity, rotation, or temperature source put on the boundary. We finally presented some application examples of the theorem in Sect. 5.

Some of the presented examples show the recovery of the cross relations that have been discussed in the literature as the Onsager-Casimir reciprocity on the basis of entropy production. It suggests some relation of the present approach to that based on the entropy production (e.g. [51,38, 19,9]). We shall discuss the issue in a separate paper, where the corollaries in Sect. 4.4 will play a key role. Here we merely stress that the present straightforward approach based on (14) is widely applicable and yields useful relations or expressions without any connection to the entropy production argument.

\section{A Reflection kernel $R$ in the linearized problem}

We summarize the properties of the reflection kernel $R\left(R_{\mathrm{CR}}\right.$ and $\left.R_{\mathrm{PR}}\right)$ for the linearized problem (see Appendix A.9 in [10]).

\section{Properties of $R_{\mathrm{CR}}$}

1. $R_{\mathrm{CR}}\left(\boldsymbol{\zeta}^{*}, \zeta ; \boldsymbol{x}\right) \geq 0$ for $\zeta_{n}^{*}<0, \zeta_{n}>0$.

2. $\int_{\zeta \cdot n>0} R_{\mathrm{CR}}\left(\boldsymbol{\zeta}^{*}, \boldsymbol{\zeta} ; \boldsymbol{x}\right) \mathrm{d} \boldsymbol{\zeta}=1 \quad$ for $\zeta_{n}^{*}<0$.

3. Let $\varphi$ be $\varphi=c_{0}+c_{i} \zeta_{i}+c_{4}|\zeta|^{2}$, where $c_{0}, c_{i}$, and $c_{4}$ are independent of $\zeta$. Among such $\varphi$, only $\varphi=c_{0}$ satisfies the relation

$$
\varphi(\boldsymbol{x}, \boldsymbol{\zeta}) E(\boldsymbol{\zeta})=\int_{\zeta_{n}^{*}<0} \frac{\left|\zeta_{n}^{*}\right|}{\left|\zeta_{n}\right|} R_{\mathrm{CR}}\left(\boldsymbol{\zeta}^{*}, \boldsymbol{\zeta} ; \boldsymbol{x}\right) \varphi\left(\boldsymbol{x}, \boldsymbol{\zeta}^{*}\right) E\left(\boldsymbol{\zeta}^{*}\right) \mathrm{d} \boldsymbol{\zeta}^{*} \quad \text { for } \zeta_{n}>0
$$

Note that the second property corresponds to the condition $\left\langle\zeta_{n} \phi\right\rangle=0$ of no flow across the boundary. The third property corresponds to the natural requirement that in a resting container with a uniform temperature the resting equilibrium state with the same temperature is established. The specular reflection is excluded from $R_{\mathrm{CR}}$ by this property.

\section{Properties of $R_{\mathrm{PR}}$}

1. $R_{\mathrm{PR}}\left(\boldsymbol{\zeta}^{*}, \zeta ; \boldsymbol{x}\right)>0$ for $\zeta_{n}^{*}<0, \zeta_{n}>0$.

2. For a certain given function $g_{0}(\boldsymbol{x}, \boldsymbol{\zeta}) \geq 0$ defined for $\zeta_{n}>0$,

$$
E(\boldsymbol{\zeta})=g_{0}(\boldsymbol{x}, \boldsymbol{\zeta})+\int_{\zeta_{n}^{*}<0} \frac{\left|\zeta_{n}^{*}\right|}{\left|\zeta_{n}\right|} R_{\mathrm{PR}}\left(\boldsymbol{\zeta}^{*}, \boldsymbol{\zeta} ; \boldsymbol{x}\right) E\left(\boldsymbol{\zeta}^{*}\right) \mathrm{d} \boldsymbol{\zeta}^{*} \text { for } \zeta_{n}>0 .
$$

3. Let $\varphi$ be $\varphi=c_{0}+c_{i} \zeta_{i}+c_{4}|\zeta|^{2}$, where $c_{0}, c_{i}$, and $c_{4}$ are independent of $\zeta$. Among such $\varphi$, only $\varphi=0$ satisfies the relation

$$
\varphi(\boldsymbol{x}, \boldsymbol{\zeta}) E(\boldsymbol{\zeta})=\int_{\zeta_{n}^{*}<0} \frac{\left|\zeta_{n}^{*}\right|}{\left|\zeta_{n}\right|} R_{\mathrm{PR}}\left(\boldsymbol{\zeta}^{*}, \boldsymbol{\zeta} ; \boldsymbol{x}\right) \varphi\left(\boldsymbol{x}, \boldsymbol{\zeta}^{*}\right) E\left(\boldsymbol{\zeta}^{*}\right) \mathrm{d} \boldsymbol{\zeta}^{*} \quad \text { for } \zeta_{n}>0
$$

The second and third properties are the counterpart to the third of $R_{\mathrm{CR}}$. They are a natural requirement that in a resting volatile container with a uniform temperature the resting equilibrium state with the same temperature and the corresponding saturation gas pressure is established. 


\section{B Condition of detailed balance}

Besides the fundamental properties in Appendix A, the kernel $R$ is often required to satisfy the so-called condition of detailed balance. For instance, the most widely used conditions, such as the diffuse reflection, Maxwell-type, and Cercignani-Lampis conditions, are known to satisfy that condition. [13]

The condition of detailed balance for the kernel $R$ in the linear problem reads

$$
\left|\zeta_{n}^{*}\right| R\left(\boldsymbol{\zeta}^{*}, \boldsymbol{\zeta} ; \boldsymbol{x}\right) E\left(\boldsymbol{\zeta}^{*}\right)=\left|\zeta_{n}\right| R\left(-\boldsymbol{\zeta},-\boldsymbol{\zeta}^{*} ; \boldsymbol{x}\right) E(\boldsymbol{\zeta}) \quad \text { for } \zeta_{n}>0, \zeta_{n}^{*}<0 .
$$

With this property, we can prove the following:

Lemma 4 Let $\phi^{A}$ and $\phi^{B}$ obey the condition (4) with $g_{\mathrm{w}}=g_{\mathrm{w}}^{A}$ and $g_{\mathrm{w}}=g_{\mathrm{w}}^{B}$ respectively. If the kernel $R$ satisfies the condition of detailed balance (31), the following equality holds:

$$
\left\langle\zeta_{n}\left(\phi^{B-}-g_{\mathrm{w}}^{B-}\right)\left(\phi^{A}-g_{\mathrm{w}}^{A}\right)\right\rangle=0 .
$$

Proof We denote $\phi^{\alpha}-g_{\mathrm{w}}^{\alpha}$ by $\psi^{\alpha}(\alpha=A, B)$ for brevity and rewrite the left-hand side as

$$
\left\langle\zeta_{n} \psi^{B-} \psi^{A}\right\rangle=\int_{\zeta_{n}>0} \zeta_{n} \psi^{B-} \psi^{A} E(\zeta) \mathrm{d} \zeta-\int_{\zeta_{n}>0} \zeta_{n} \psi^{B} \psi^{A-} E(\zeta) \mathrm{d} \zeta
$$

The first term is rewritten by the use of (4) for $\psi^{A}$ as

$$
\int_{\zeta_{n}>0} \zeta_{n} \psi^{B-} \psi^{A} E(\boldsymbol{\zeta}) \mathrm{d} \boldsymbol{\zeta}=\int_{\zeta_{n}>0}\left[\int_{\zeta_{n}^{*}<0}\left|\zeta_{n}^{*}\right| R\left(\boldsymbol{\zeta}^{*}, \boldsymbol{\zeta} ; \boldsymbol{x}\right) E\left(\boldsymbol{\zeta}^{*}\right) \psi^{A *} \mathrm{~d} \boldsymbol{\zeta}^{*}\right] \psi^{B-} \mathrm{d} \boldsymbol{\zeta}
$$

while the second term is rewritten as

$$
\begin{aligned}
\int_{\zeta_{n}>0} \zeta_{n} \psi^{B} \psi^{A-} E(\boldsymbol{\zeta}) \mathrm{d} \boldsymbol{\zeta} & =\int_{\zeta_{n}>0}\left[\int_{\zeta_{n}^{*}<0}\left|\boldsymbol{\zeta}_{n}^{*}\right| R\left(\boldsymbol{\zeta}^{*}, \boldsymbol{\zeta} ; \boldsymbol{x}\right) E\left(\boldsymbol{\zeta}^{*}\right) \psi^{B *} \mathrm{~d} \boldsymbol{\zeta}^{*}\right] \psi^{A-} \mathrm{d} \boldsymbol{\zeta} \\
& =\int_{\zeta_{n}^{*}<0}\left[\int_{\zeta_{n}>0}\left|\zeta_{n}\right| R\left(-\boldsymbol{\zeta},-\boldsymbol{\zeta}^{*} ; \boldsymbol{x}\right) E(\boldsymbol{\zeta}) \psi^{B-} \mathrm{d} \boldsymbol{\zeta}\right] \psi^{A *} \mathrm{~d} \boldsymbol{\zeta}^{*}
\end{aligned}
$$

Thus, the first and second terms cancel out each other because of the detailed balance (31).

Acknowledgements This work is supported in part by KAKENHI (No. 19560066) from JSPS and by the research grant from the UBE Foundation. This work was motivated by the interest in the Onsager reciprocity that the author shared with Professors Yoshio Sone and Tai-Ping Liu. The author expresses his sincere thanks to Professor Sone for his encouragement, helpful discussions, and comments on the manuscript. This work was finished during the stay at the Institute of Mathematics, Academia Sinica. The author expresses his sincere thanks to Professor Liu for the hospitality and his continuing interest in this work.

\section{References}

1. E. H. Kennard, Kinetic Theory of Gases, McGraw-Hill, New York (1938)

2. S. K. Loyalka, Kinetic theory of thermal transpiration and mechanocaloric effect. I, J. Chem. Phys. 55, 4497-4503 (1971).

3. S.P. Bakanov, V.V. Vysotskij, B.V. Deryaguin, and V.I. Roldughin, Thermal polarization of bodies in the rarefied flow, J. NonEquilib. Thermodyn. 8, 75-83 (1983).

4. S. Takata, Y. Sone, and K. Aoki, Numerical analysis of a uniform flow of a rarefied gas past a sphere on the basis of the Boltzmann equation for hard-sphere molecules, Phys. Fluids A 5, 716-737 (1993).

5. P. S. Epstein, Zur Theorie des Radiometer, Z. Phys. 54, 537-563 (1929).

6. Y. Sone, Flow induced by thermal stress in rarefied gas, Phys. Fluids 15, 1418-1423 (1972).

7. S. Takata, K. Aoki, and Y. Sone, Thermophoresis of a sphere with a uniform temperature: Numerical analysis of the Boltzmann equation for hard-sphere molecules, in Rarefied Gas Dynamics, B. D. Shizgal and D. P. Weaver eds. (AIAA, Washington, D. C., 1994), pp. 626-639.

8. F. Sharipov, Onsager-Casimir reciprocity relations for open gaseous systems at arbitrary rarefaction. II. Application of the theory for single gas, Physica A 203, 457-485 (1994). 
9. V. M. Zhdanov and V. I. Roldughin, Non-equilibrium thermodynamics and kinetic theory of rarefied gases, Physics Uspekhi 41, 349-378 (1998).

10. Y. Sone, Molecular Gas Dynamics, Birkhäuser, Boston (2007); Supplementary Notes and Errata: Kyoto University Research Information Repository (http://hdl.handle.net/2433/66098).

11. Y. Sone, Asymptotic theory of flow of rarefied gas over a smooth boundary I, in Rarefied Gas Dynamics, L. Trilling and H. Y. Wachman, eds. (Academic Press, New York, 1969), Vol. I, pp. 243-253.

12. Y. Sone, Kinetic Theory and Fluid Dynamics, Birkhäuser, Boston (2002); Supplementary Notes and Errata: Kyoto University Research Information Repository (http://hdl.handle.net/2433/66099).

13. C. Cercignani, The Boltzmann Equation and Its Application, Sect. III.3, Springer-Verlag, New York (1988).

14. T. -P. Liu and S. -H. Yu, The Green's function and large-time behavior of solutions for the onedimensional Boltzmann equation, Commun. Pure Appl. Math. 57, 1543-1608 (2004).

15. S. Sunakawa, Theoretical Elecrtomagnetism, Kinokuniya, Tokyo (1999). (in Japanese)

16. J. Jeans, The Mathematical Theory of Electricity and Magnetism, 5th ed. reprinted, Cambridge University Press, New York (1960).

17. W. K. H. Panofsky and M. Phillips, Classical Electricity and Magnetism, 2nd ed. reprinted, AddisonWesley, Tokyo (1962).

18. S. Chandrasekhar, Radiative Transfer, Dover, New York (1960).

19. F. Sharipov, Onsager-Casimir reciprocity relations for open gaseous systems at arbitrary rarefaction. I. General theory for single gas, Physica A 203, 437-456 (1994).

20. F. Sharipov, Onsager-Casimir reciprocal relations based on the Boltzmann equation and gas-surface interaction: Single gas, Phys. Rev. E 73, 026110 (2006).

21. F. R. W. McCourt, J. J. M. Beenakker, W. E. Köhler, and I. Kuščer, Nonequilibrium Phenomena in Polyatomic Gases, Vol. 2, Chapt. 16, Clarendon Press, Oxford (1991).

22. H. Grad, Singular and nonuniform limits of solutions of the Boltzmann equation, in Transport Theory, R. Bellman, G. Birkhoff, and I. Abu-Shumays, eds. (AMS, Providence, 1969), pp. 269-308

23. I. Imai, Some applications of function theory to fluid dynamics, in 2nd Intern. JSME Symp. Fluid Mechinery and Fluidics, Tokyo, pp. 15-23 (1972).

24. T. Tatsumi, Fluid Mechanics, Baifukan, Tokyo (1982). (in Japanese)

25. J. Happel and H. Brenner, Low Reynolds Number Hydrodynamics, 2nd ed., Noordhoff International Publishing, Leyden (1973).

26. S. L. Sobolev, Partial Differential Equations of Mathematical Physics, Dover, New York (1989).

27. Y. Sone, Thermal creep in rarefied gas, J. Phys. Soc. Jpn 21, 1836-1837 (1966).

28. T. Ohwada, Y. Sone, and K. Aoki, Numerical analysis of the shear and thermal creep flows of a rarefied gas over a plane wall on the basis of the linearized Boltzmann equation for hard-sphere molecules, Phys. Fluids A 1, 1588-1599 (1989).

29. C. E. Siewert, Viscous-slip, thermal-slip, and temperature jump coefficients as defined by the linearized Boltzmann equation and the Cercignani-Lampis boundary condition, Phys. Fluids 15, 1696-1701 (2003).

30. N. B. Maslova, Kramers problem in the kinetic theory of gases, USSR Comp. Math. Phys. 22, 208-219 (1982).

31. C. Bardos, R. E. Caflisch, and B. Nicolaenko, The Milne and Kramers problems for the Boltzmann equation of a hard sphere gas, Comm. Pure Appl. Math. 39, 323-352 (1986).

32. F. Coron, F. Golse and C. Sulem, A classification of well-posed kinetic layer problems, Commun. Pure Appl. Math. 41, 409-435 (1988).

33. F. Golse and F. Poupaud, Stationary solutions of the linearized Boltzmann equation in a half-space, Math. Methods Appl. Sci. 11, 483-502 (1989).

34. Y. Sone and Y. Onishi, Kinetic theory of evaporation and condensation - Hydrodynamic equation and slip boundary condition, J. Phys. Soc. Jpn 44, 1981-1994 (1978).

35. S. Yasuda, S. Takata, and K. Aoki, Numerical analysis of the shear flow of a binary mixture of hardsphere gases over a plane wall, Phys. Fluids 16, 1989-2003 (2004).

36. Y. P. Pao, Application of kinetic theory to the problem of evaporation and condensation, Phys. Fluids $\mathbf{1 4}$ 306-312 (1971).

37. Y. Sone, T. Ohwada, and K. Aoki, Evaporation and condensation on a plane condensed phase: Numerical analysis of the linearized Boltzmann equation for hard-sphere molecules, Phys. Fluids A 1, 1398-1405 (1989).

38. D. Bedeaux, L.J.F. Hermans, and T. Ytrehus, Slow evaporation and condensation, Physica A 169, 263 280 (1990).

39. Y. Sone, T. Ohwada, and K. Aoki, Temperature jump and Knudsen layer in a rarefied gas over a plane wall: Numerical analysis of the linearized Boltzmann equation for hard-sphere molecules, Phys. Fluids A 1, 363-370 (1989). 
40. S. Yasuda, S. Takata, and K. Aoki, Evaporation and condensation of a binary mixture of vapors on a plane condensed phase: Numerical analysis of the linearized Boltzmann equation, Phys. Fluids 17, 047105 (2005).

41. S. Takata, S. Yasuda, K. Aoki, and S. Kosuge, Temperature, pressure, and concentration jumps for a binary mixture of vapors on a plane condensed phase: Numerical analysis of the linearized Boltzmann equation, Phys. Fluids 18, 067102 (2006).

42. V.G. Chernyak and A.Ye. Margilevskiy, The kinetic theory of heat and mass transfer from a spherical particle in a rarefied gas, Int. J. Heat Mass Transfer. 32, 2127-2134 (1989).

43. S. Takata, Y. Sone, D. Lhuillier, and M. Wakabayashi, Evaporation from or condensation onto a sphere: Numerical analysis of the Boltzmann equation for hard-sphere molecules, Computers Math. Applic. 35, 193-214 (1998).

44. S. Takata and Y. Sone, Flow induced around a sphere with a non-uniform surface temperature in a rarefied gas, with application to the drag and thermal force problems of a spherical particle with an arbitrary thermal conductivity, Eur. J. Mech. B/Fluids 14, 487-518 (1995).

45. T. Ohwada, Y. Sone, and K. Aoki, Numerical analysis of the Poiseuille and thermal transpiration flows between two parallel plates on the basis of the Boltzmann equation for hard-sphere molecules, Phys. Fluids A 1, 2042-2049 (1989).

46. Y. Sone, S. Takata, and T. Ohwada, Numerical analysis of the plane Couette flow of a rarefied gas on the basis of the linearized Boltzmann equation for hard-sphere molecules, Eur. J. Mech. B/Fluids 9, 273-288 (1990).

47. R. D. M. Garcia and C. E. Siewert, The linearized Boltzmann equation with Cercignani-Lampis boundary conditions: Basic flow problems in a plane channel, Eur. J. Mech. B/Fluids 28, 387-396 (2009).

48. Y. Sone, Y. Waniguchi, and K. Aoki, One-way flow of a rarefied gas induced in a channel with a periodic temperature distribution, Phys. Fluids 8, 2227-2235 (1996).

49. M. Knudsen, Thermischer Molekulardruck der Gase in Röhren, Ann. Phys. (Leipzig) 33, 1435-1448 (1910).

50. H. Sugimoto and Y. Sone, Vacuum pump without a moving part driven by thermal edge flow, in Rarefied Gas Dynamics, M. Capitelli ed. (AIP, Melville, New York, 2005), pp. 168-173.

51. D. Bedeaux, Nonequilibrium thermodynamics and statistical physics of surfaces, in Advances in Chemical Physics, I. Prigogine and S.A. Rice eds., Vol. LXIV, John-Wiley \& Sons, New York, 47-109 (1986). 